BNL-96818-2011-IR

C-A/AP/\#441

Dec. 2011

\title{
Analysis of Vernier Scans during the PP2PP run in 2009 (pp at $100 \mathrm{GeV} / \mathrm{beam})$
}

\author{
A. Drees
}

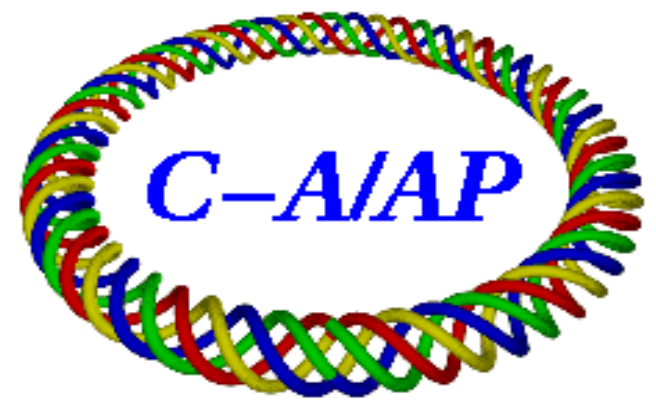

\section{Collider-Accelerator Department Brookhaven National Laboratory Upton, NY 11973}

Notice: This document has been authorized by employees of Brookhaven Science Associates, LLC under Contract No. DE-AC02-98CH10886 with the U.S. Department of En ergy. The United States Government retains a nonexclusive, paid-up, irrevocable, world-wide license to publish or reproduce the published form of this document, or allow others to do so, for United States Government purposes. 


\title{
Analysis of Vernier Scans during the PP2PP run in 2009 (pp at $100 \mathrm{GeV} /$ beam)
}

\author{
A. Drees \\ December 13, 2011
}

\section{Introduction}

At the end of RHIC's 2009 operation a dedicated run for the PP2PP experiment (part of the STAR experiment) took place from Jun 29 to Jul 06 2009. Polarized protons were accelerated to $100 \mathrm{GeV}$ using ramp-file pp100-90pp2pp with a $\beta^{*}=22 \mathrm{~m}$ in IR6. Since only transverse polarization was required no rotator ramp was in use. The PP2PP experiment consists mainly of two Roman Pot detectors (one horizontal and one vertical) on either side of IR6 in the outgoing-beam arms between the Q3 and Q4 magnets. The yellow pots are in sector 5 , the blue ones in sector 6 . Roman Pot type detectors are installed inside the beampipe causing an accelerator safety concern. To address this concern there is a limit to the allowable total beam current in the machine while Roman Pots are enabled to move closer to the beam. This limit was set to a motion limit of $5 \mathrm{~mm}$ from the center of the beampipe and $50 \cdot 10^{11}$ beam current per ring [1]. In order to reduce the background in the detectors, beams were scraped using the RHIC collimator system prior to moving the pots closer. This was typically repeated several times throughout a store since beam halo reforms over the course of hours.

\section{Vernier Scan Technique}

During the dedicated PP2PP run in 2009 a total of 3 vernier scans [2] were performed. The transverse size and shape of the beam overlap region is measured by recording the interaction rate as a function of the transverse beam separation. A Gauss-fit of the measured interaction rate as a function of the separation allows to determine the effective beam size as well as the maximum achievable collision rate and the effective cross section of the detector in use (see eq. 1). Typically additional effects such as a possible crossing angle or the hourglass effect require correction factors to be applied. However, at a large value of $\beta^{*}=22 \mathrm{~m}$, which was used for this dedicated run, these effects are negligible while others, such as errors in position measurements, need to be considered carefully. Table 1 lists the fills with vernier scans, the fill pattern and the beam current in units of number of protons.

From the fit to the vernier scan data the RMS beam size $\sigma_{x, y}$ as well as the effective cross section $\sigma_{B B C}^{e f f}$ of a detector, in this case the STAR BBCs, can be derived:

$$
\sigma_{B B C}^{e f f}=\frac{R_{\text {max }} 2 \pi n_{B} n_{Y} \sigma_{x}^{V S} \sigma_{y}^{V S}}{n_{\text {coll }} f_{\text {rev }} N_{B} N_{Y}}
$$




\begin{tabular}{|l|c|c|c|c|c|}
\hline fill & ring & date & time & fill pattern & \# coll. pairs \\
\hline 11020 & Y & $07-01-09$ & $04: 49-05: 33$ & $90 \times 89$ & 63 \\
11031 & Y & 07-03-09 & 19:08- 19:30 & $90 \times 90$ & 64 \\
11032 & Y & $07-04-09$ & $03: 23-03: 45$ & $90 \times 90$ & 64 \\
\hline
\end{tabular}

Table 1: List of fills with vernier scans during the 2009 PP2PP run.

where:

$R_{\text {max }}=$ maximum collision rate seen by the BBC detector (corrected for background)

$n_{B}, n_{Y}=$ number of blue and yellow bunches respectively

$\sigma_{x, y}^{V S}=$ RMS beam-overlap size, derived from the fit to the vernier scan data

$n_{\text {coll }}=$ number of colliding bunch pairs in the IP where the BBC detector is located

$f_{\text {rev }}=$ revolution frequency, approx. $78.4 \mathrm{kHz}$

$N_{B, Y}=$ total number of blue and yellow protons

Given the RMS beam size we can calculate the normalized beam emittance $\epsilon_{\text {norm }}^{x, y}$ assuming that the beam sizes are the same in the two rings:

$$
\epsilon_{\text {norm }}^{x, y}=\frac{6\left(\sigma_{x, y}^{V S}\right)^{2} \gamma}{2 \beta^{*}}
$$

with $\gamma=106.8$ for $100 \mathrm{GeV}$ protons.

\section{The Data}

According to equation 1, the effective detector cross section can be determined by the beam current, the collision rate and the overlap region of the two beams. Beam position monitors are the major contributors in determining the overlap region and define the associated systematic errors.

\subsection{Beam Current Measurements}

Even though the beam decay for pp2pp running is reduced to $2 \%$ per hour or better, there is still a small slope to the beam current as a function of time. The amount of protons lost during the course of the vernier scans is summarized in table 2. Fig. 1 shows 4 histograms

\begin{tabular}{|l|c|c|c|c|c|c|c|c|}
\hline fill & \multicolumn{2}{|c|}{$I_{D C C T}^{\text {blue }}\left[10^{11}\right]$} & \multicolumn{2}{|c|}{$I_{D C C T}^{\text {yell }}\left[10^{11}\right]$} & \multicolumn{2}{c|}{ protons lost } & \multicolumn{2}{c|}{ DCCT-WCM } \\
& mean & RMS & mean & RMS & blue & yell & blue & yell \\
\hline 11020 & 40.3 & 0.02 & 45.2 & 0.01 & $310^{9}$ & $1310^{9}$ & $-0.25 \%$ & $+1 \%$ \\
11031 & 44.1 & 0.01 & 44.6 & 0.015 & $410^{9}$ & $510^{9}$ & $+0.36 \%$ & $+0.4 \%$ \\
11032 & 45.4 & 0.015 & 42.7 & 0.02 & $610^{9}$ & $910^{9}$ & $-0.6 \%$ & $+0.03 \%$ \\
\hline
\end{tabular}

Table 2: Beam current measurements during the pp2pp vernier scans.

containing the beam current measurements in the two rings using the two available devices, 
$\operatorname{DCCT}$ and WCM ( [3]). Units are number of protons $* 10^{11}$. Similar figures for the two other stores with vernier scans (11031 shown in figure 12 and 11032 shown in figure 13) are added in the appendix for reference. The measured beam loss is typically about the same for the DCCT and WCM. Since the numbers quoted in table 2 correspond to an insignificant loss over the course of a vernier scan $(0.3 \%$ or less), a constant is a fair approximation for the beam current during the scan. The non-gaussian shape apparent in

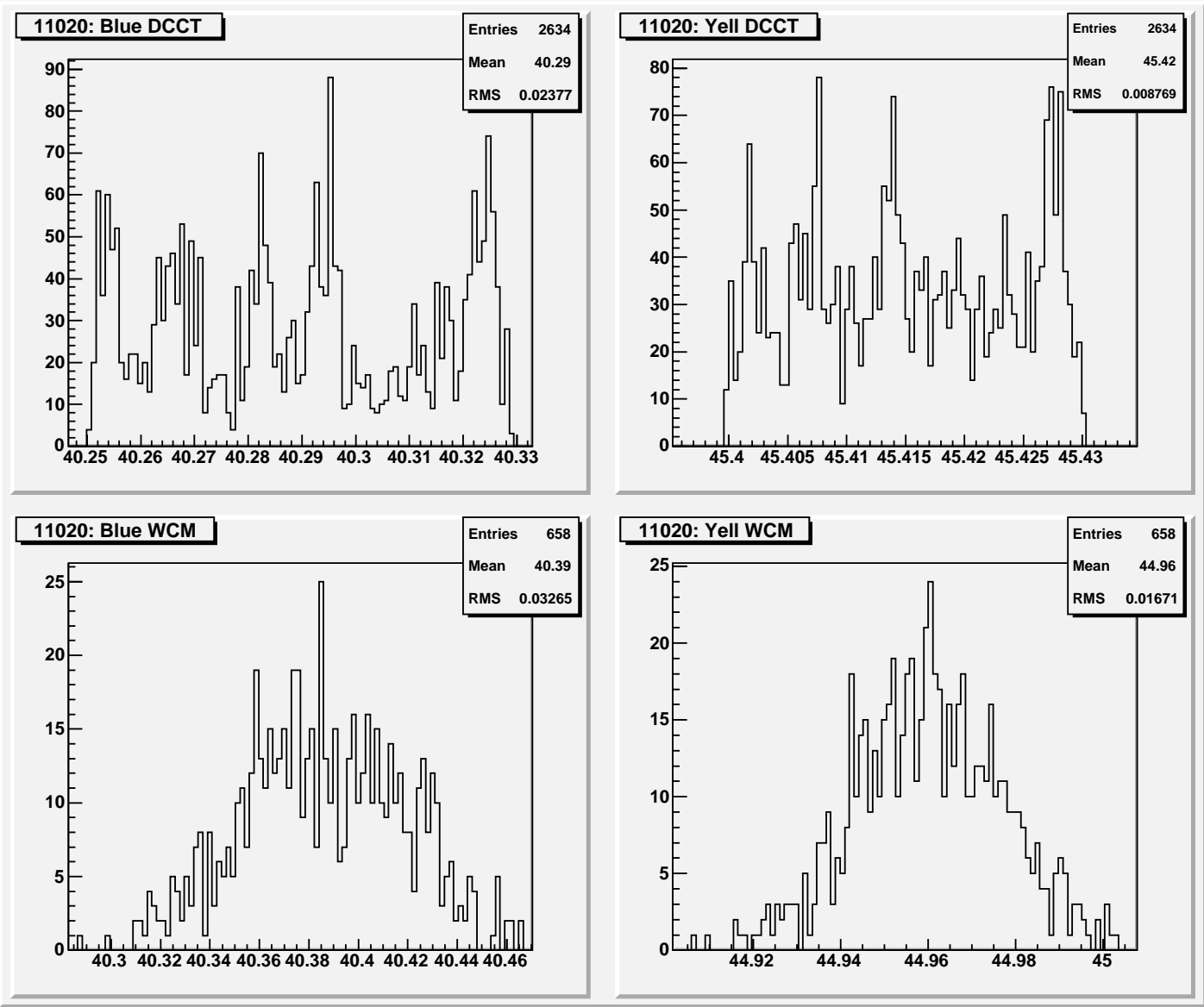

Figure 1: Beam Current measurements during the vernier scan in fill 11020.

the DCCT data is an artifact of the slope, however small, still being larger than the typical scatter of the measurements. Therefore the quoted RMS is dominated by the drop in beam current over the duration of the scan. Since only one value per scan is used to calculate the cross section, this RMS represents the correct "scatter" to be used in the analysis although it underestimates the devices accuracy. The relative difference in percent between the two instruments (DCCT and WCM) is also summarized in table 2. A negative difference indicates the WCM readings being larger than the DCCT readings. Since the DCCT is the more accurate device $(0.2 \%$ accuracy, [3]) and debunching is typically insignificant when RHIC is operated with protons, the DCCT values ("Mean" value in the graphs in figures 1, 12 and 13) will be used in the analysis with the corresponding RMS values and an added overall $1 \%$ systematic error per beam to take into account remaining measurement uncertainties associated with beam current measurements. 


\subsection{Beam Position Measurements}

The DX BPMs are dual-plane BPMs, located at $-8.325 \mathrm{~m}$ (Sec 5 ) and $+7.99 \mathrm{~m}$ (Sec 6) away from the center of IR6. They are the only BPMs installed within the common beam pipe and are used for both, blue and yellow beam position measurements. The beam signal from the DX BPM striplines generates a double pulse with a leading positive pulse for beam traveling in one direction and a doublet with a negative leading pulse for beam traveling in the opposite direction. The stripline analog signal is split and fed into two independent electronic modules, each having a dedicated acquisition trigger timed to the pulse corresponding to the appropriate ring. Figure 2 illustrates the two beam, double pulse concept (from [4]). Shown are the signals from 3 bunches, two bunches in

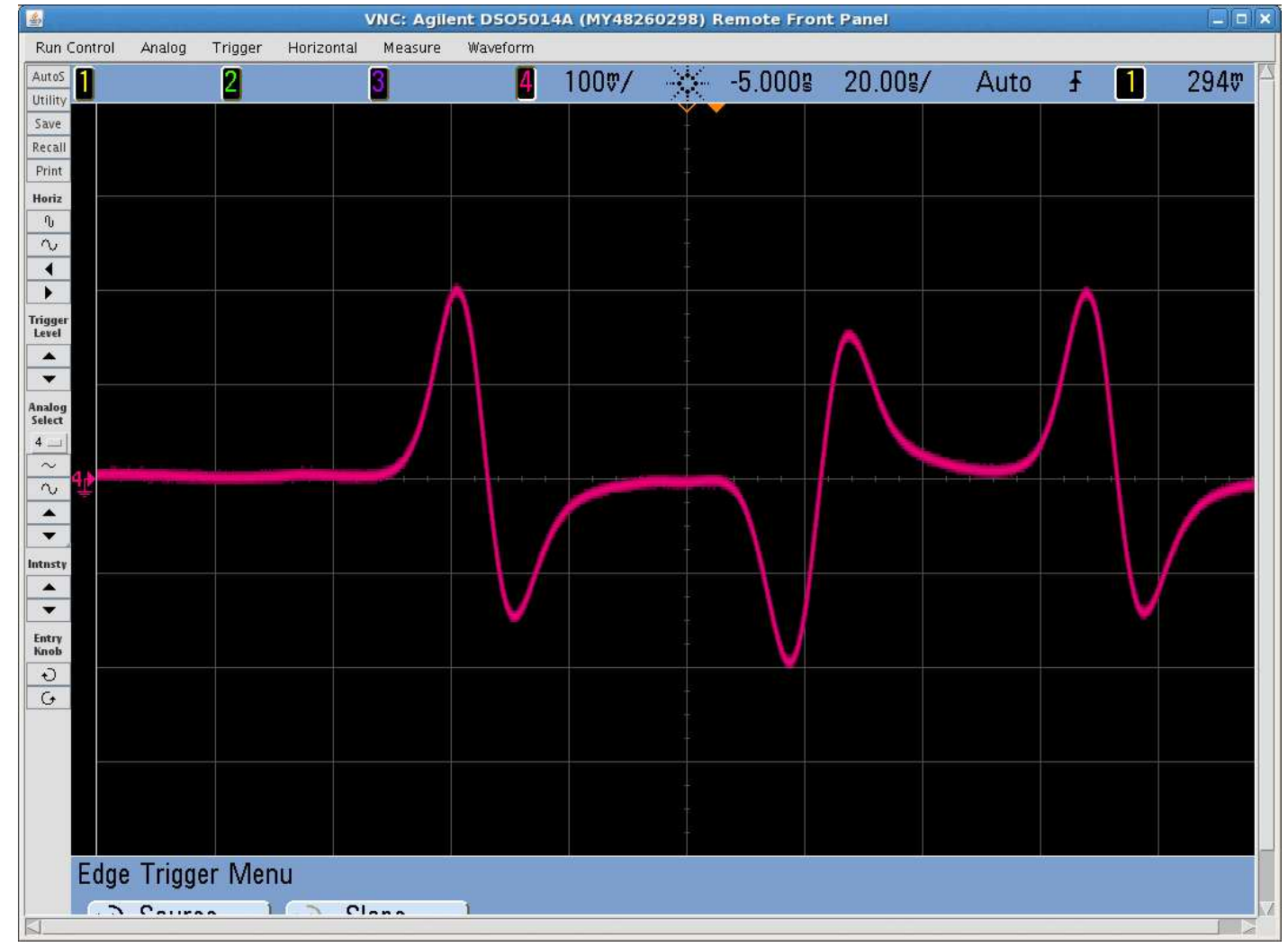

Figure 2: Scope image depicting the raw signal from an IP8 DX BPM, Jun 222011.

one direction and one bunch in the opposite direction. The two leading positive pulses are approximately $110 \mathrm{~ns}$ apart, consistent with a 120 bunch pattern. The signal from the beam traveling the opposite direction, identifiable by the leading negative pulse, falls right between the other two bunch signals.

Due to the large value of $\beta^{*}=22 \mathrm{~m}$ in the STAR IR for the pp2pp run the RMS beam-overlap size grew from about $200 \mu \mathrm{m}$ (at a $\beta^{*}=0.7 \mathrm{~m}$ ) to about $1 \mathrm{~mm}$. Therefore, the vernier scan had to cover much more transverse displacement than usual, namely $\pm 3.5 \mathrm{~mm}$, a total of $7 \mathrm{~mm}$. Figure 3 shows the measured horizontal beam position, seen by the two individual DX-BPMs on either side of the IR, as a function of the requested or "set" displacement (from online-model). The top two graphs show the Blue DX BPMs in Sector 5 (left) and in Sector 6 (right). The bottom two graphs contain the Yellow DX BPM measurements. Since the scan was done by moving the yellow beam rather than the blue, 

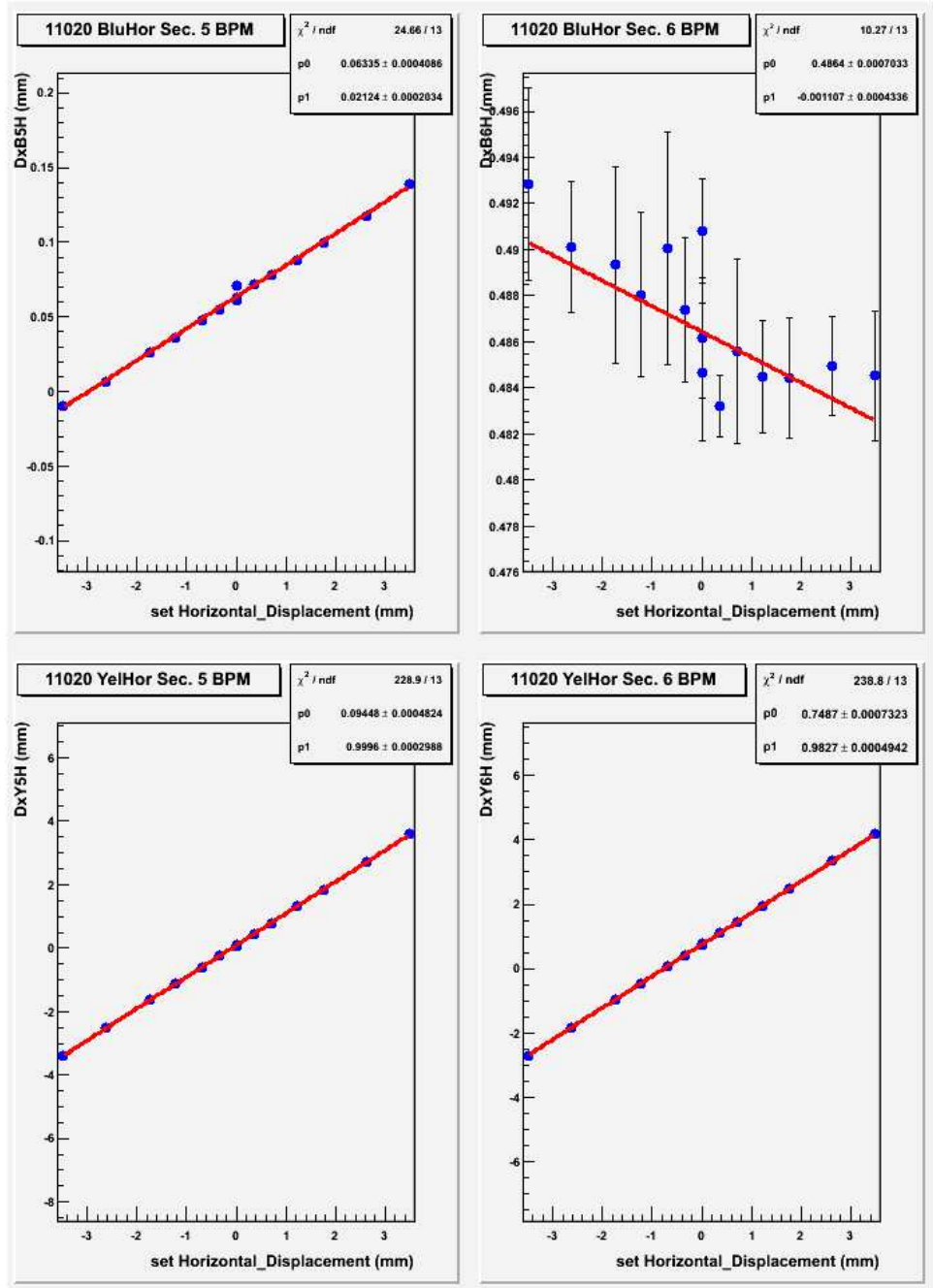

Figure 3: Horizontal BPMs during horizontal vernier scan 11020.

we expect to see a slope of $\mathrm{p} 1=1$ for the yellow BPMs and, more or less, no measurable movement in the Blue BPMs. The equivalent graphs from the other plane combinations are added in the appendix for the records. Figure 14 shows the vertical readings during the horizontal scan, figure 15 presents the vertical readings during the vertical scan and figure 16 shows the horizontal data during the vertical scan. Similar data was obtained for the 2 other scans though it is now shown here.

In figure 3 the yellow horizontal BPM measurements (bottom two plots) agree to better than $1 \%$ with the set value. It appears that the blue BPM DxB5H (top left) measures approximately $2 \%=150 \mu \mathrm{m}$ of the $7 \mathrm{~mm}$ motion while there is no significant measurement of motion in DxB6H $(\leq 10 \mu m)$. Table 3 summarizes the average BPM readings for a 1 $\mathrm{mm}$ set value during the 6 scans ( 3 horizontal and 3 vertical). The average reading is obtained from a linear fit to the data as applied and shown in figures 3 and figure 14 to figure 16. Values below $0.1 \%$ are not taken into account but are considered consistent with "zero".

The signals are combined according to equation 3 in chapter 4 . Looking at the values 


\begin{tabular}{|l||c|c|c||c|c|c|}
\hline \multicolumn{1}{|l||}{ BPM } & \multicolumn{3}{c||}{ Horizontal } & \multicolumn{3}{c|}{ Vertical } \\
& 11020 & 11031 & 11032 & 11020 & 11031 & 11032 \\
\hline \hline DxB5H & 0.021 & 0.022 & 0.022 & - & - & - \\
DxB6H & -0.001 & 0.007 & 0.004 & - & - & - \\
blue H comb. & 0.010 & 0.014 & 0.013 & - & - & - \\
\hline DxY5H & 1.0 & 0.986 & 0.987 & 0.006 & 0.006 & 0.006 \\
DxY6H & 0.983 & 1.025 & 1.023 & -0.022 & -0.021 & -0.022 \\
yell H comb. & $\mathbf{0 . 9 9 2}$ & $\mathbf{1 . 0 0 5}$ & $\mathbf{1 . 0 0 5}$ & -0.008 & -0.008 & -0.008 \\
\hline \hline DxB5V & - & - & - & 0.012 & 0.011 & 0.010 \\
DxB6V & - & - & - & 0.022 & 0.034 & 0.020 \\
blue V comb. & - & - & - & 0.017 & 0.028 & 0.015 \\
\hline DxY5V & -0.003 & - & -0.002 & 0.939 & 0.928 & 0.927 \\
DxY6V & 0.015 & 0.016 & 0.015 & 0.952 & 0.997 & 0.998 \\
yell V comb. & 0.006 & 0.008 & 0.007 & $\mathbf{0 . 9 4 5}$ & $\mathbf{0 . 9 6 2}$ & $\mathbf{0 . 9 6 2}$ \\
\hline
\end{tabular}

Table 3: BPM read-back for a $1 \mathrm{~mm}$ set value during the 3 vernier scans. Values below $0.1 \%$ are not considered.

printed in bold it can be concluded that the set values (i.e. the online model) and BPM read-backs are in perfect agreement for the horizontal plane while for the vertical plane there seems to be an averaged $4.4 \%$ mismatch, i.e. the BPMs consistently read about $40 \mu \mathrm{m}$ short of a $1 \mathrm{~mm}$ steering request. This is significantly more than the typical RMS scatter of $2.5 \mu \mathrm{m}$ to $5.5 \mu \mathrm{m}$ of BPM readings during a randomly chosen 30 minute period at store during the dedicated pp2pp operation (see figures 17 and 18 in Appendix). Since the averaged $4.4 \%$ mismatch is consistent for the three stores, the set values for the vertical plane should be corrected with no correction applied in the horizontal plane. Before applying any correction, however, we will compare this measurement with the Q1 $\mathrm{BPM}$ readings (see chapter 3.2.1 below). A possible correction as well as a systematic error will be determined based on the results from both devices.

An important question remains: do we have to consider the apparent cross-talk or coupling between the two planes (transverse) and the two rings? It is conceivable that changing one beams position by $7 \mathrm{~mm}$ affects the position of the other beam. At the same time it is possible that, due to local coupling, a bump in the horizontal beam position 'leaks' into the vertical plane and vice versa. However, electronic cross-talk between the striplines would look like coupling without any real beam motion. A small shift in timing or ringing might appear like a small signal from the opposite ring. Readings from the DxB5H and DxB6H BPMs during the horizontal scan could be both, real motion or a fake signal from the opposite ring electronic modules. The blue DxB5H consistently sees about $2 \%$ of the yellow beam motion while $\mathrm{DxB} 6 \mathrm{H}$ does not detect any changes. However, a real beam motion should cause a signal on both sides of the IR. A similar argument applies to the yellow vertical readings during the horizontal scans. While they are consistent from store to store they only appear on one side (DxY6V), the combined effect at the vertex location (extrapolated to the IR between the two BPMs) is small and below 1\%. Likewise 
the readings in the horizontal plane during the vertical scans appear mostly on one side only $(\mathrm{DxY} 6 \mathrm{H})$ and the combined effect is small and below 1\%. These results indicate that any transverse coupling, if real and not electronic, is small. We concluded similarly for the coupling between the rings. The blue vertical signals during the yellow vertical scans are between $1 \%$ and $3 \%$ and appear on both sides, the combined effect varies from store to store and is between $1.5 \%$ and $2.8 \%$. However, there should be no reason why any alleged coupling into the opposite ring would be larger in one plane than the other, indicating there is no real ring-to-ring coupling. This notion is confirmed when analyzing Q1 BPM data and difference orbits (see chapter 3.2.1 and 3.2.2 below).

\subsubsection{Comparison with Q1 BPMs}

In order to obtain additional coupling information we looked at the yellow Q1 BPMs as well as the entire ring. The ring BPMs, in particular the arc BPMs have the advantage that they are single plane (i.e. no electronic coupling between the striplines from the two planes) and all but the DX BPMs are for one ring only (i.e. no electronic cross-talk between the two rings possible). Figure 4 contains the Q1 BPM data from the vertical section of the 11032 vernier scan. Note that the Q1 BPM are dual plane BPMs just as the Dx BPMs and electronic coupling between the striplines is certainly a possibility for both. The error for Q1 BPM readings is set to $5 \mu \mathrm{m}$ in the plots.

\begin{tabular}{|l||c|c|c||c|c|c|}
\hline HOR 1 mm & yo5-bh1 & yi6-bh1 & comb. & yo5-bv1 & yi6-bv1 & comb. \\
\hline 11020 & 1.032 & 0.974 & 1.003 & 0.004 & -0.001 & 0.002 \\
\hline 11031 & 1.019 & 0.973 & 0.996 & 0.006 & 0.0 & 0.003 \\
\hline 11032 & 1.021 & 0.968 & 0.993 & 0.004 & 0.0 & 0.002 \\
\hline & DxY5H & DxY6H & comb. & DxY5V & DxY6V & comb. \\
\hline 11020 & 1.00 & 0.983 & 0.992 & -0.003 & 0.015 & 0.006 \\
\hline \hline VER $\mathbf{1} \mathbf{~ m m}$ & yo5-bh1 & yi6-bh1 & comb. & yo5-bv1 & yi6-bv1 & comb. \\
\hline 11020 & 0.008 & -0.017 & -0.005 & 0.911 & 1.028 & 0.970 \\
\hline 11031 & 0.009 & -0.017 & -0.004 & 0.913 & 1.031 & 0.972 \\
\hline 11032 & 0.008 & -0.017 & -0.005 & 0.915 & 1.028 & 0.971 \\
\hline & DxY5H & DxY6H & comb. & DxY5V & DxY6V & comb. \\
\hline 11020 & 0.006 & -0.022 & -0.008 & 0.939 & 0.952 & 0.945 \\
\hline
\end{tabular}

Table 4: Q1 BPM readings for a $1 \mathrm{~mm}$ set value during both, the horizontal and the vertical sections of the three vernier scans. The readings from the Dx BPMs in scan 11020 are copied from table 3 and added for convenience.

Table 4 summarizes the results from analyzing the Q1 BPM data. The top half shows the measurements of the horizontal (left) and vertical (right) Q1 BPMs when a horizontal bump of $1 \mathrm{~mm}$ is applied. All three scans are listed and the equivalent measurements of the DxBPMs from one scan are added for reference at the bottom. The bottom half sows the equivalent results for a vertical $1 \mathrm{~mm}$ bump. Looking at the horizontal data and comparing it with the results in table 3 paints a quite consistent picture. Requested 


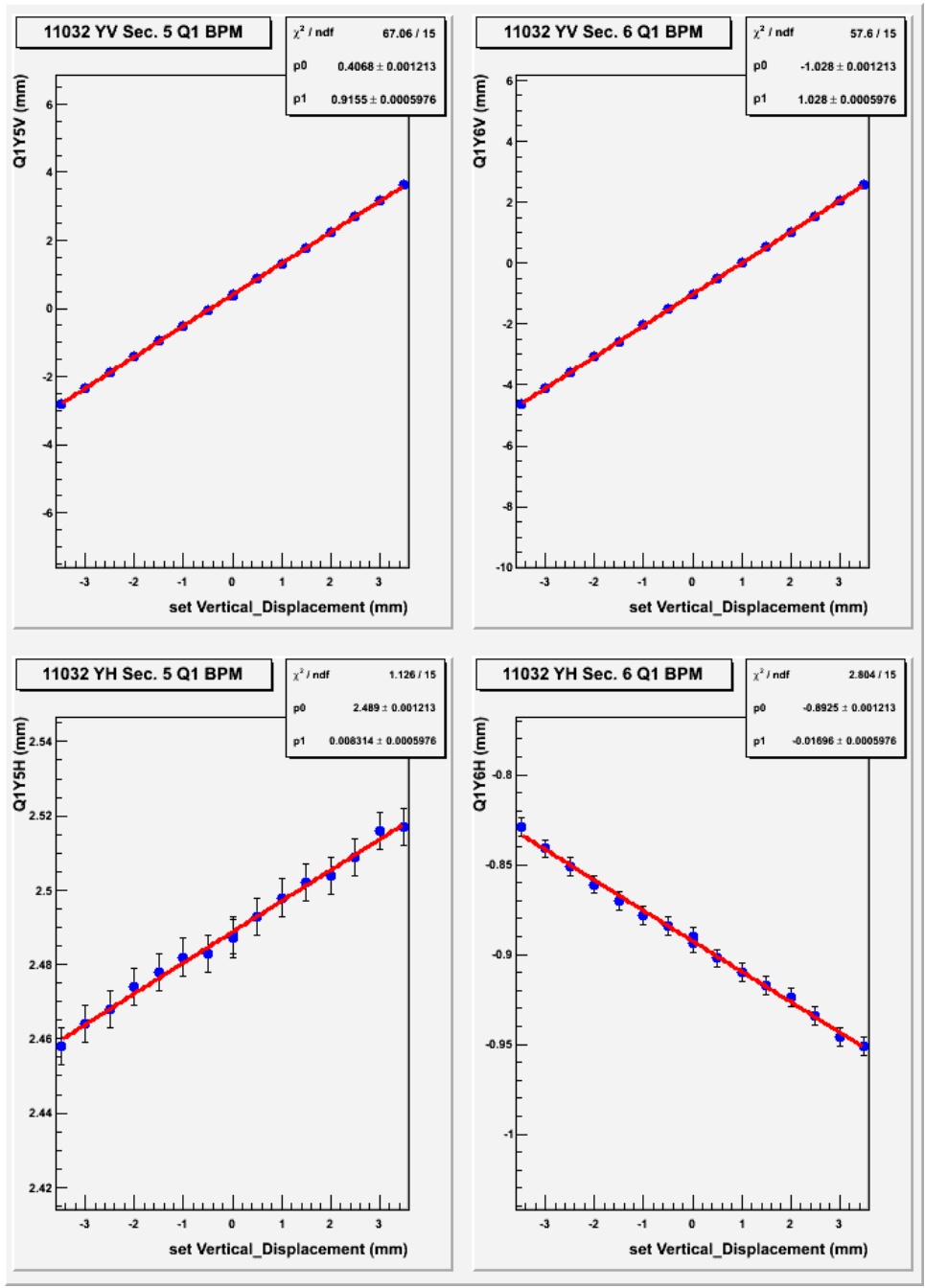

Figure 4: Vertical (top) and horizontal (bottom) Q1 BPMs during the vertical vernier scan 11032 .

and measured motion agree to better than 1\% using both types of BPMs. The Q1 BPMs confirm the presence of a very small horizontal angle of about $1 \mu \mathrm{rad}$. Such a small angle is of no concern, in particular not when operating with a large $\beta^{*}$. There is very little evidence of coupling from the horizontal into the vertical plane, in fact the small signal of $-0.3 \%$ seen by DxY5V is matched by its Q1 BPM counterpart y05-bv1 $(+0.4 \%)$ but with opposite sign! The $+1.5 \%$ coupling seen by DxY6V is not confirmed by the Q1 BPM yi6-bv1 which sees a very small $-0.1 \%$ effect and with opposite sign as well. This indicates that the alleged 'coupling' is not a beam effect but electronic coupling instead since any beam based effect should be visible in both, the DX BPM as well as the Q1 BPM. It also implies, however, that electronic coupling apparently can amount to $1.5 \%$ of the motion in the other plane and any signal of that size in an opposite plane could be attributed to an electronic effect, if it is confirmed by another BPM - also subject to electronic coupling - or not!

The comparison between the Q1 and Dx BPMs for the vertical scan gives slightly 
different results. A stronger coupling into the sector 6 side, which could indicate an angle, as seen by DxY6H is indeed confirmed by the Q1 BPM and points to coupling from the vertical plane into the horizontal (while there is no sign of coupling from the horizontal plane into the vertical). However, an electronic effect present in both BPM types (see above) cannot be excluded with the presented data. In any case, the combined effect, extrapolated to the interaction point, shows an effect of less than $1 \%$ using both BPM types. Both BPM types agree on a small vertical angle with the same sign of about 2 $\mu \mathrm{rad}$ in all stores. All results in both planes are consistent for all three scans. Thus an additional systematic error of $1 \%$ is assumed for coupling between the planes while no correction is applied.

Combining the Q1 BPM position measurements of the 3 scans in the vertical plane results in an averaged undershoot of $-2.9 \%$ instead of the $-4.4 \%$, seen by the Dx BPMs, when compared with the requested motion. This small discrepancy between the two devices is likely due to different gains and possibly differences in BPM calibrations. Since both devices, Q1 and Dx BPMs agree on the measured position being smaller than the requested (set) value, a correction should be applied. Thus the vertical position will be corrected by $-3.6 \%$, combining both Q1 and Dx BPM measurements, and a systematic error of $1.5 \%$ is added to the other BPM related systematic errors.

\subsubsection{Global Orbit Measurements}

When checking the entire ring we looked at the difference orbits of two orbits taken 10 minutes apart outside any intentional beam activity to have a baseline for comparison. The RMS of such a difference orbit reflects the measurement reproducibility and turns out to be about $5 \mu \mathrm{m}$. Computing difference orbits in the opposite ring (blue) while the yellow displacement was set to $+3.5 \mathrm{~mm}$ and $-3.5 \mathrm{~mm}$ respectively results in a global RMS in the opposite ring of twice to three times the baseline, about 10-18 $\mu \mathrm{m}$ indicating that there is indeed some beam motion 'leaking' into the other ring. The effect is the same for the two planes. This indicates that an alleged 'leak' into the opposite ring is measurable but insignificant. Based on this and the results above no correction for ring-to-ring cross-talk is applied and a systematic error of $1 \%$ added to cover the associated uncertainties.

However, the RMS of the difference orbit in the same ring but opposite plane results in an effect of about 6 times as much as the coupling into the opposite ring as shown below in figure 5 which contains two examples of difference orbits. The green dots represent a difference orbit of two vertical orbits taken at the $-2.625 \mathrm{~mm}$ step and the $-3.5 \mathrm{~mm}$ step

$Y$ orbit

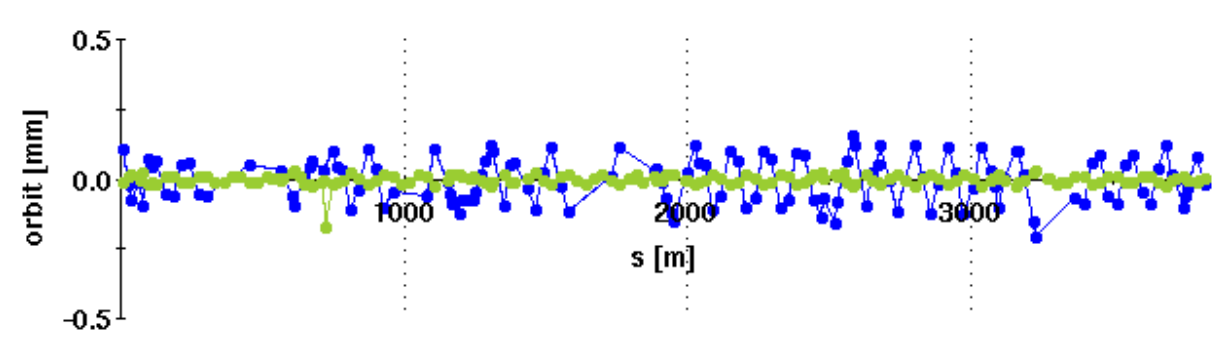

Figure 5: Difference Orbits in the yellow vertical plane taken at various steps during the horizontal scan in fill 11020. 
during the horizontal scan. The difference in the horizontal position accounts to $875 \mu \mathrm{m}$, approximately $10 \%$ of the full range of motion $(7 \mathrm{~mm})$. The RMS of the difference orbit (arcs only) comes out to be $14 \mu \mathrm{m}$, about three times more than the baseline but still insignificant. The blue dots represent a difference orbit of two vertical orbits taken at the $+3.5 \mathrm{~mm}$ and $-3.5 \mathrm{~mm}$ step during the horizontal scan. The difference in the horizontal position at the IR is at its maximum of $7 \mathrm{~mm}$. The RMS of this difference orbit amounts to about $80 \mu \mathrm{m}$. There is no variation with inclusion or exclusion of the straight sections from the RMS calculation. This alludes to other areas than the straight sections as the culprit for the coupling, caused by a non-closure of the horizontal 4-bump. The RMS of the difference orbits in the horizontal plane (excluding the straight sections) is twice the size of the RMS of the vertical difference orbits. Analyzing difference orbits in the horizontal plane during the vertical part of the scan gives similar results. The difference orbit computed using the $+3.5 \mathrm{~mm}$ and $-3.5 \mathrm{~mm}$ settings has an $80 \mu \mathrm{m}$ RMS. However, the RMS in the vertical plane itself (excluding the straight sections) is about 4 times this, almost $300 \mu \mathrm{m}$, indicating a larger 'leak' of the 4-bump in the vertical plane. These 'leaks', however, are of no real concern for the analysis of the vernier scans. Inclusion of the straight section into the RMS calculation of the horizontal difference orbits increases the RMS by $30 \%$.

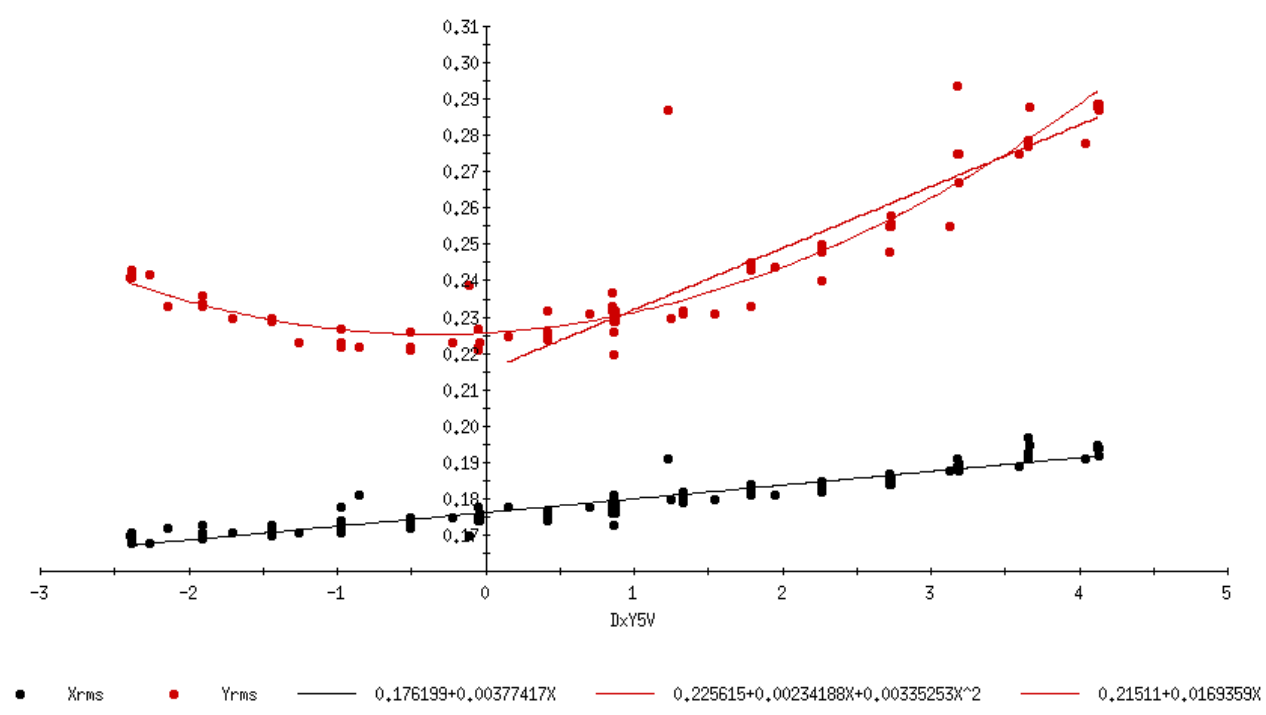

Figure 6: Yellow Yrms and Xrms measurements as a function of vertical displacement during the vertical vernier scan in fill 11032.

Figure 6 illustrates another means to observe coupling into the opposite plane. The red data points correspond to the vertical global orbit RMS, Yrms, varying during the course of the vertical vernier scan in fill 11032 with a minimum somewhere around 0 and growing towards either side. The black dots, labeled Xrms, correspond to the global orbit RMS in the other plane, here the horizontal one. The total change in Xrms is small but still visible and clearly correlated with the scan. It totals about $15 \mu \mathrm{m}$. The equivalent range in the vertical plane is, as can be expected, larger (about $70 \mu \mathrm{m}$ ) and indicates the amount of closure of the applied 4-bumps, depending on the size of that bump.

Considering all available data, no correction for a beam motion in the opposite ring 
will be applied since a beam based cross-talk between the rings appears insignificant. In addition, coupling between the planes is apparent but small at IR6. It cannot be excluded that most of the observed local coupling is due to electronic cross-talk between the striplines of the dual plane BPMs. Thus, no correction will be applied but a systematic error of $1 \%$ for transverse coupling, as quoted earlier, is confirmed and added.

\subsection{Collision Rate}

Identical ZDC detectors [5] are installed as luminosity or collision rate monitors at each IR. They are the monitor of choice for book-keeping purposes because of low background conditions and the fact that more or less identical detectors are available at each interaction region. However, due to the DX magnets in front of them, the only particles from the interaction region they are exposed to are uncharged particles, photons and neutrons. Thus the effective cross section of the ZDCs is much smaller than the BBC cross section for proton running. Running with a $\beta^{*}=22 \mathrm{~m}$ reduces the collision rate for the ZDCs even further to a point where the number of collisions seen in the ZDCs is too small to provide a measurement with enough statistical accuracy. Figure 7 illustrates the difference

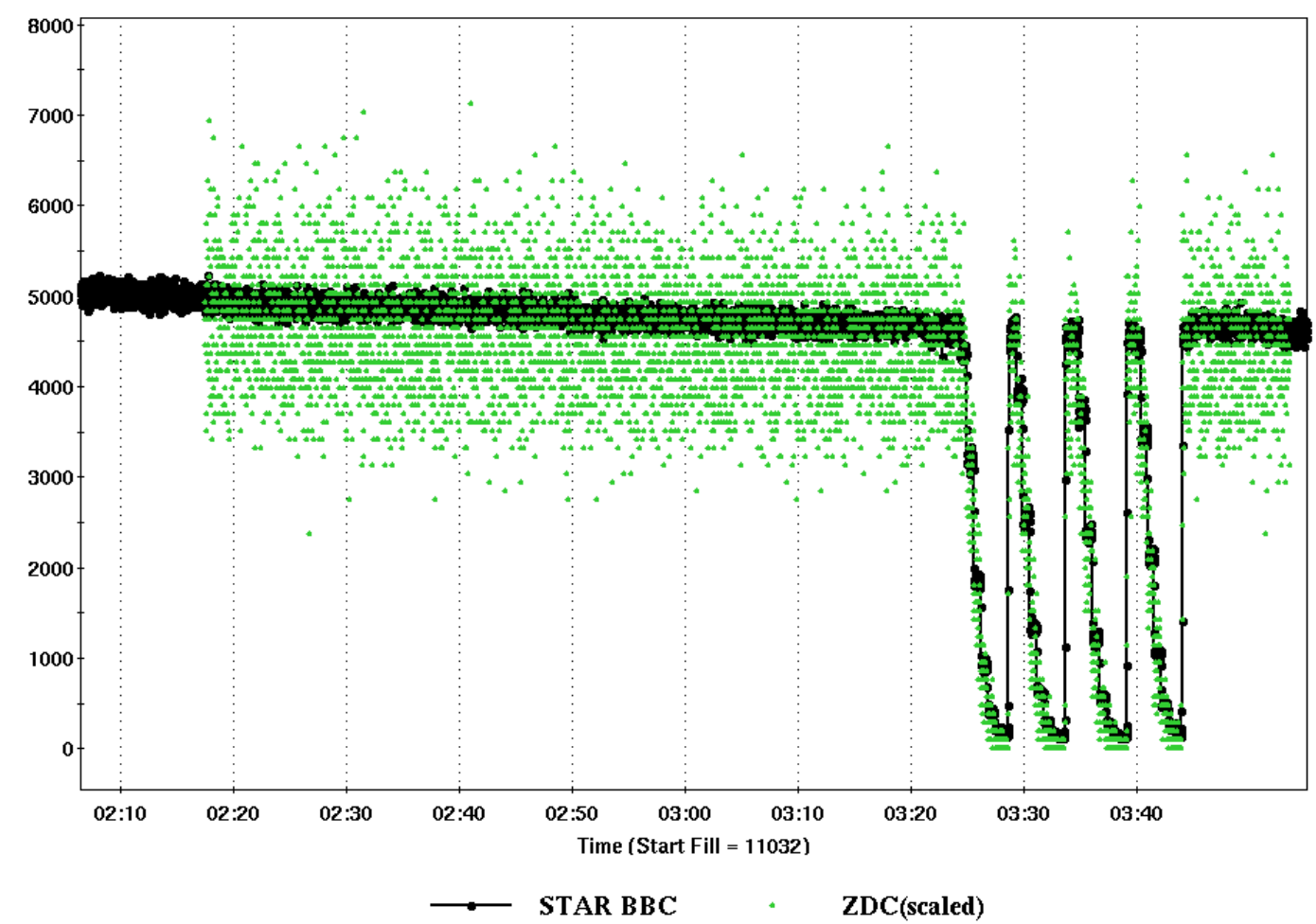

Figure 7: Collision rate as a function of time in store 11032 as seen by the BBC (black dots) and the ZDC (green stars). The ZDC readings are scaled with a factor 95.

in statistical significance of the two detectors. The ratio of the two cross sections is approximately 95-100. In figure 7 the ZDC readings are scaled with a factor 95 , for figure 8 they were scaled with a factor 98 . Figure 7 emphasizes the ZDC peak-to-peak scatter that is apparently a factor of about 10 larger than the scatter of the BBC measurements, as 


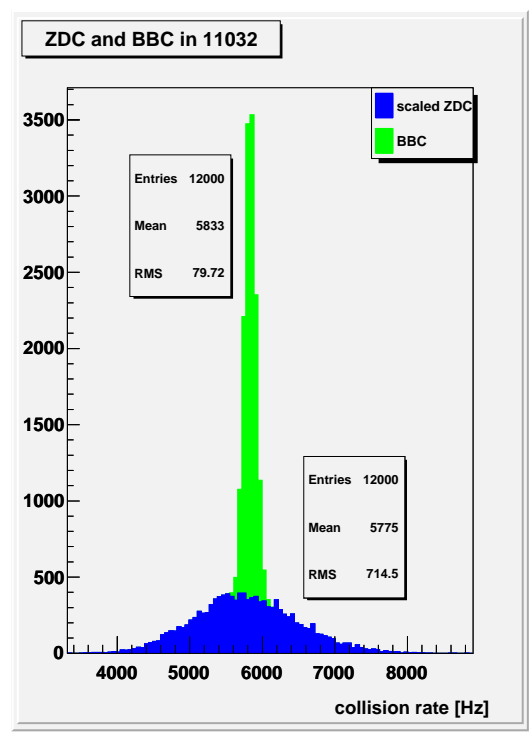

Figure 8: A histogram of the collision rates from $3.3 \mathrm{~h}$ during store 11032 as seen by the $\mathrm{BBC}$ (green) and ZDC (blue) detectors. The ZDC is scaled with a factor 98 and both are slope corrected to emphasize the different RMS.

can be expected. After correcting both signals for the slope, i.e. the luminosity lifetime, they can be directly compared in a histogram, shown in figure 8 while obliterating the effect from luminosity lifetime. The RMS of the ZDC readings is in fact a factor 9 larger than the RMS of the BBC measurements. Therefore, for this analysis, we will use the $\mathrm{BBC}$ detector to monitor collision rates and determine luminosity.

\section{$4 \quad$ Vernier Scan Results}

\subsection{Cross Section}

As pointed out above, the BBC detectors are used for this analysis. Graphs showing data from 5 out of the total of 6 scans, 3 horizontal and 2 vertical, are attached in the appendix (figures 19 to 23) for reference. One data-set is presented below in figure 9 as a "show and tell" example. All data sets are presented in two graphs, collision rates as a function of set displacement (i.e. model) at the top, and collision rates as a function of measured displacement at the bottom. The measured displacement $d_{\text {meas }}$ uses the read-back from the two DX BPMs on either side of the IP, interpolated to the center of the IR using the surveyed distance from the center:

$$
d_{\text {meas }}=(8.325 m \cdot D x Y 5 \mathcal{P}+7.99 m \cdot D x Y 6 \mathcal{P}) /(16.315 m)
$$

with the plane $\mathcal{P}$ either $\mathrm{H}$ (horizontal) or $\mathrm{V}$ (vertical). The $4 \%$ difference between the two BPM locations (see chapter 3.2), where the BPM in sector 5 is about $0.34 \mathrm{~m}$ closer to the IP than the sector $6 \mathrm{BPM}$, is taken into account.

The $\chi^{2} / n d f$ in this example is about 1 ( 1 or smaller for the other scans, see appendix) and practically the same for the two approaches. Note that the set value is corrected by a factor 0.964 as described above in chapter 3.2. All fit parameters (offset, max and 


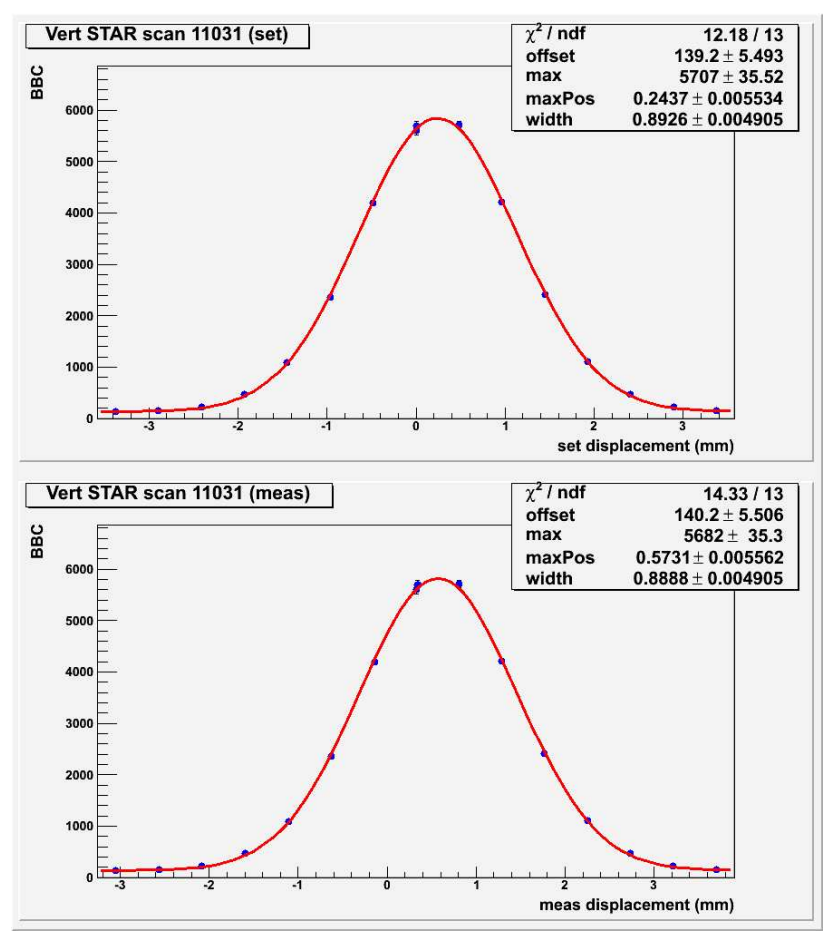

Figure 9: Vertical vernier scan at IR6. Shown is the collision rate from the BBC as a function of the displacement using the set values (top) and the measured values (bottom). Only statistical errors are used in the graph.

width) with the exception of "maxPos" agree to a level of better than $1 \%$ and are identical within the error bars. The position of the maximum, "maxPos", is meaningless for the approach using the set displacement. The set value defines the starting position to be " 0 " by default. Thus the "maxPos" fit parameter announces the initial deviation of the two colliding bunches from perfect head-on collisions (in this case approx. $240 \mu \mathrm{m}$ ) at the time when the vernier scan is started.

\begin{tabular}{|l|c|c|c|c|}
\hline fill & $\begin{array}{c}\sigma_{B B C}^{\text {eff }} \\
{[\mathrm{mbarn}]}\end{array}$ & $\begin{array}{c}\text { inst. } \mathcal{L} \\
{\left[10^{29} \mathrm{~cm}^{-2} s^{-1}\right]}\end{array}$ & $\begin{array}{c}\sigma_{x}^{V S} \\
{[\mathrm{~mm}]}\end{array}$ & $\begin{array}{c}\sigma_{y}^{V S} \\
{[\mathrm{~mm}]}\end{array}$ \\
\hline 11020 & $24.0 \pm 0.5$ & $1.20 \pm 0.03$ & $1.23 \pm 0.01$ & $1.21 \pm 0.01$ \\
11031 & $23.9 \pm 0.3$ & $2.36 \pm 0.03$ & $0.92 \pm 0.01$ & $0.89 \pm 0.01$ \\
11032 & $23.4 \pm 0.3$ & $1.95 \pm 0.03$ & $1.00 \pm 0.01$ & $0.98 \pm 0.01$ \\
\hline
\end{tabular}

Table 5: Results from the 32009 pp2pp vernier scans. Quoted errors are statistical only.

Table 5 summarizes the results from the 3 scans. The cross section $\sigma_{B B C}^{e f f}$ is calculated using equation 1 . The $\mathrm{BBC}$ rates $R_{B B C}$ were corrected for accidental coincidences using:

$$
R_{B B C}^{\text {corr }}=R_{B B C}-\frac{R_{B B C}^{B l u e} \cdot R_{B B C}^{Y \text { ell }}}{n_{\text {coll }} \cdot f_{\text {rev }}}
$$


where

$R_{B B C}$ is the uncorrected $\mathrm{BBC}$ collision rate, $R_{B B C}^{B l u e, Y e l l}$ is the rate seen by a single side of the BBC detector, $n_{\text {coll }}$ is the number of colliding bunch pairs in IR6 and

$f_{\text {rev }}$ is the revolution frequency.

This correction is very small and does not exceed $1 \%$ for any of the 3 scans. A systematic error of $1 \%$ is added for the singles correction to cover remaining uncertainties. Combining the 3 scans the effective cross section for the BBC detector results in:

$$
\sigma_{\mathrm{BBC}}^{\mathrm{eff}}=23.7 \pm 0.2 \text { (stat.) mbarn }
$$

as shown in figure 10 . The $\chi^{2} / n d f=1$ indicates that the variation of the three measurements is consistent with their statistical error bars.

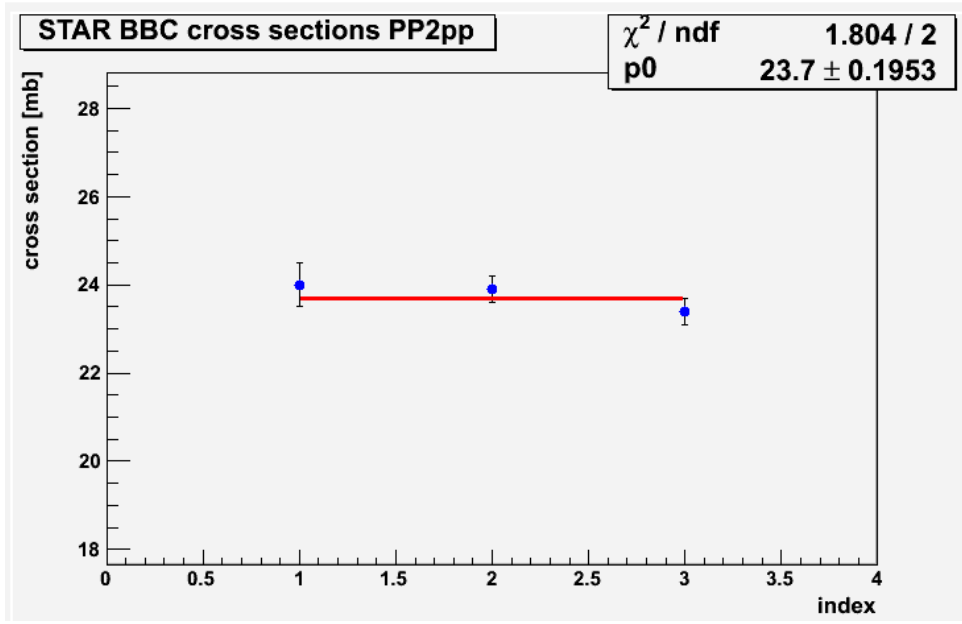

Figure 10: Summary of the BBC effective cross sections, measured by vernier scans.

\subsection{Emittance}

The beam size, assuming the same size for the blue and yellow beams, can be obtained from the fit parameters ("width") and is listed in table 5. The normalized emittance $\epsilon_{x, y}$ is calculated according to equation 2 and summarized in table 6 . The size of the betafunction at the location of the measurement (IR6) is a needed input parameter and the value $\beta^{*}=22 m$ from the ramp-file $p p 100-90 p p 2 p p$ is used to obtain the results in table 6 . A $10 \%$ error is assigned to the emittance measurements to cover statistical and systematic uncertainties such as the measured width and possible beta-beating at the IR, known to be easily as large as $10 \%$. The emittance from the vernier scans is compared to RHIC IPM readings. While a scan is in progress the IPM takes 2 measurements per minute resulting in about 40-80 readings in the same time interval. Since there is no significant increase of emittance for a time period of 20 to 30 minutes a constant is a fair approximation for the emittance at the time of the scan. The quoted values in table 6 represent the mean values for that time span. The measurements from the two rings are combined into one value to be compared with the result from the vernier scan. The quoted errors are the RMS of the scatter around this mean as shown in figure 11. Store 11020 was chosen as a representative example. The IPM detector face a similar problem with the value of the 


\begin{tabular}{|l|c|c|c|c|}
\hline fill & $\begin{array}{c}\epsilon_{x}^{V S} \\
{[\pi \mathrm{mm} \mathrm{mrad}]}\end{array}$ & $\begin{array}{c}\epsilon_{y}^{V S} \\
{[\pi \mathrm{mm} \mathrm{mrad}]}\end{array}$ & $\begin{array}{c}\epsilon_{x}^{I P M} \\
{[\pi \mathrm{mm} \mathrm{mrad}]}\end{array}$ & $\begin{array}{c}\epsilon_{y}^{I P M} \\
{[\pi \mathrm{mm} \mathrm{mrad}]}\end{array}$ \\
\hline 11020 & $22 \pm 2$ & $21 \pm 2$ & $18 \pm 2$ & $20 \pm 2$ \\
11031 & $12 \pm 1$ & $12 \pm 1$ & $11 \pm 2$ & $11 \pm 2$ \\
11032 & $15 \pm 2$ & $14 \pm 1$ & $13 \pm 2$ & $13 \pm 2$ \\
\hline
\end{tabular}

Table 6: Emittance measurements for 3 pp2pp stores from vernier scans and the RHIC IPMs.

beta-function at the location of the IPM detectors. The value there could just as well differ by 10 to $20 \%$ from the value obtained from the model. However, the two measurements
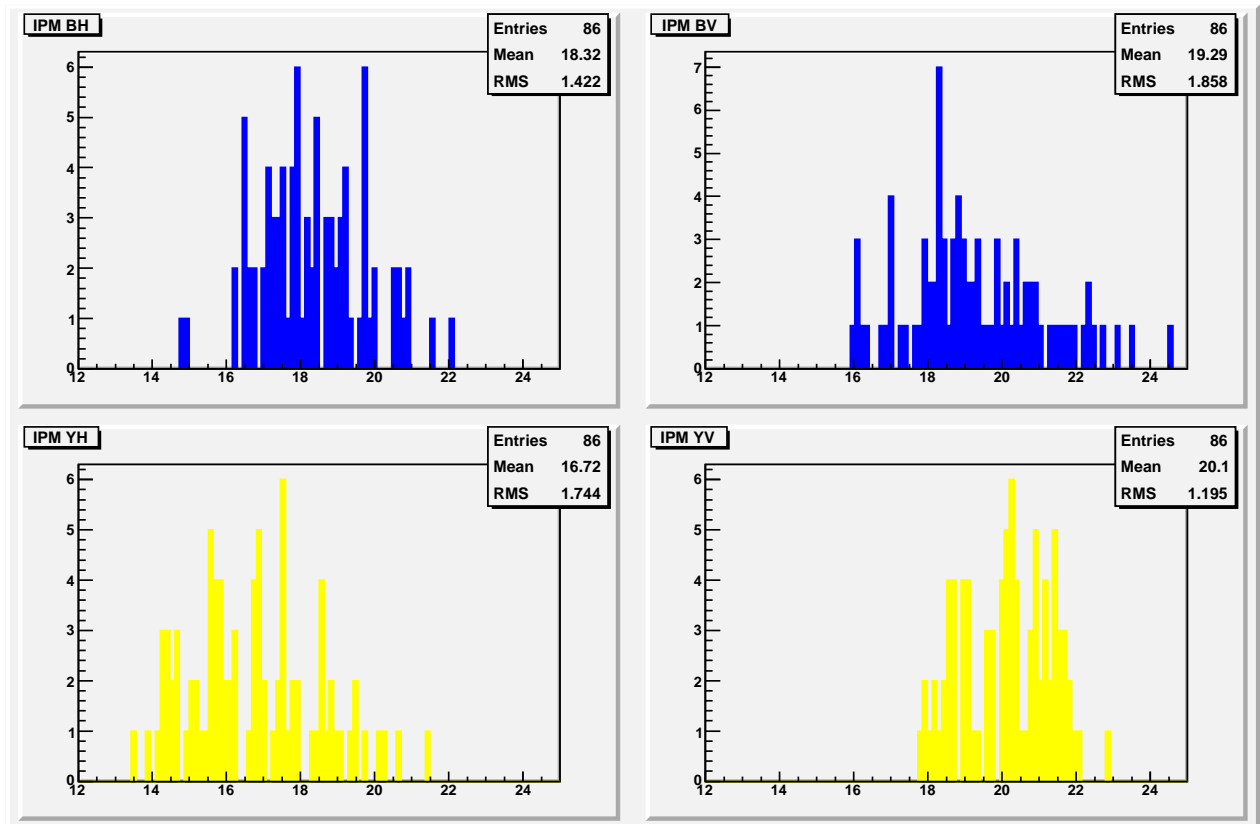

Figure 11: IPM emittance measurement readings from the time interval of the vernier scan in store 11020 .

show a respectable agreement for all three stores within the quoted errors, indicating that the beta-beat when operating with lattice pp100-90pp2pp is insignificant.

\section{Summary}

Combining the 3 scans the effective cross section for the BBC detector results in:

$$
\sigma_{B B C}^{e f f}=23.6 \pm 0.2 \text { (stat.) } \pm 0.6 \text { (syst.) mbarn }
$$

Systematic errors for beam current measurements (1\%), model vs. measured beam position $(1.5 \%)$, transverse coupling $(1 \%)$, ring-to-ring cross-talk $(1 \%)$ and singles correction $(1 \%)$ are taken into account. The combined systematic errors add up to a total of $2.5 \%$. 
Observed emittances were between 12 and $22 \pi \mathrm{mm}$ mrad with beam sizes between $600 \mu \mathrm{m}$ and $900 \mu \mathrm{m}$.

\section{References}

[1] C-A TPL 09-09, "TEMPORARY PROCEDURE FOR RHIC OPERATION WITH ROMAN POTS"

[2] S. Van Der Meer, ISR-PO/68-31, KEK68-64.

[3] http://www.cadops.bnl.gov/Instrumentation/InstWiki/index.php/ RHIC_Current_Transformer

[4] R. Michnoff, the BPM_2011 systems elog, Wed Jun 22 2011, 16:21:58.

[5] A. Baltz et al., Nucl. Instr. and Methods, A417 (1998) 1 


\section{Appendix: Graphs}
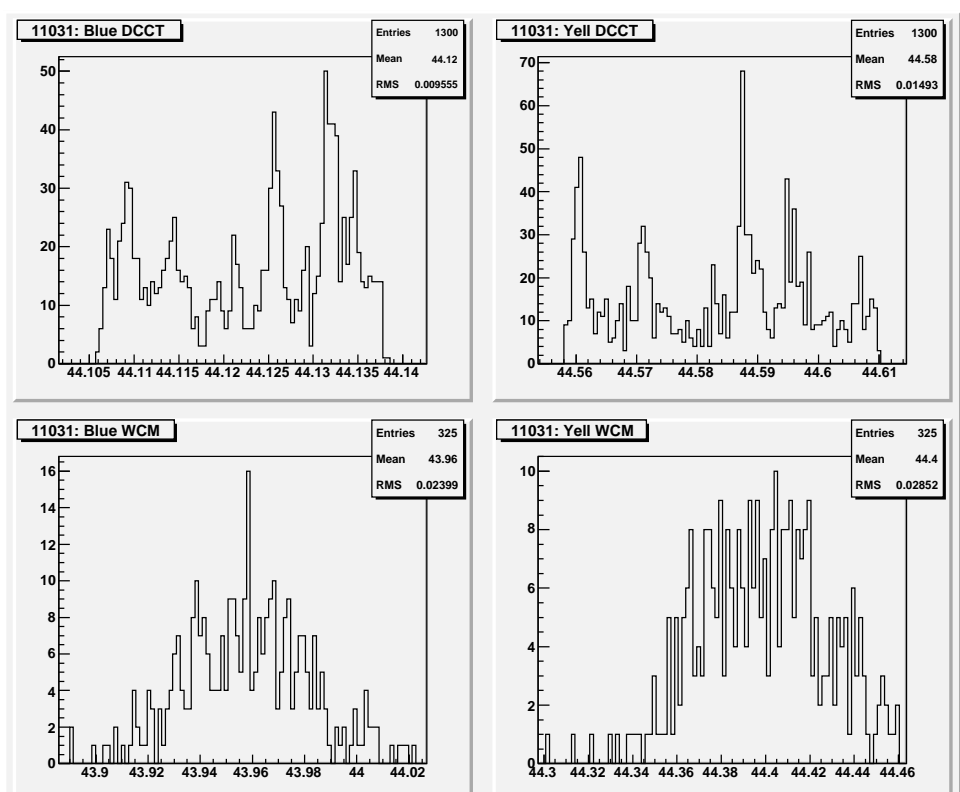

Figure 12: Beam Current measurements during the vernier scan in fill 11031.

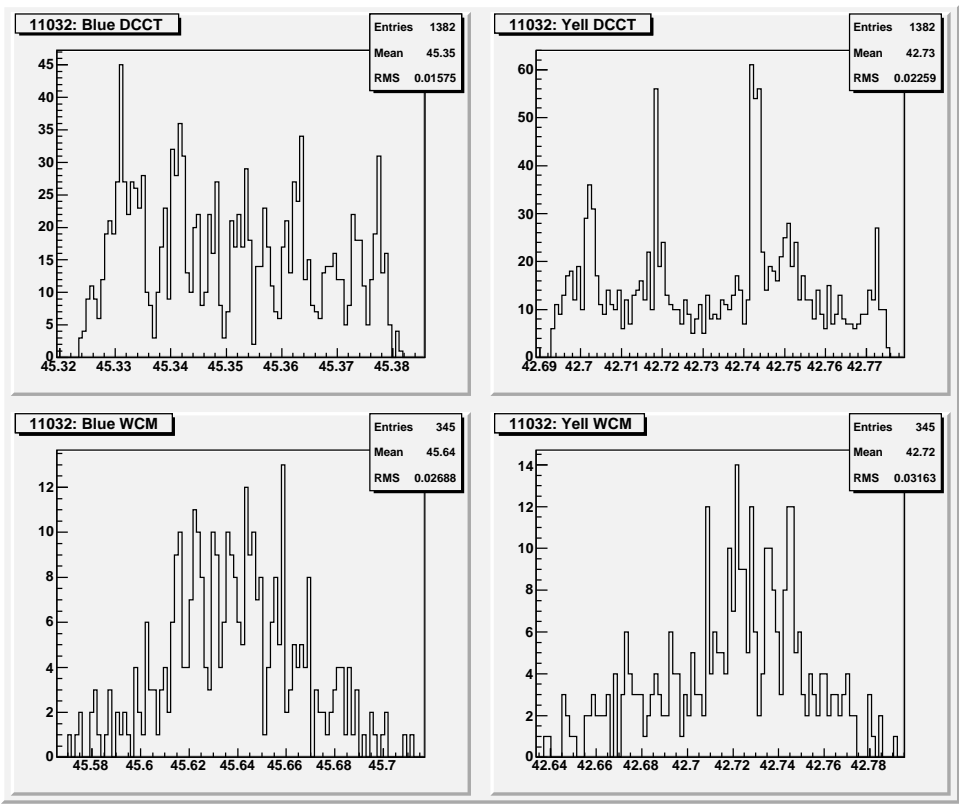

Figure 13: Beam Current measurements during the vernier scan in fill 11032. 

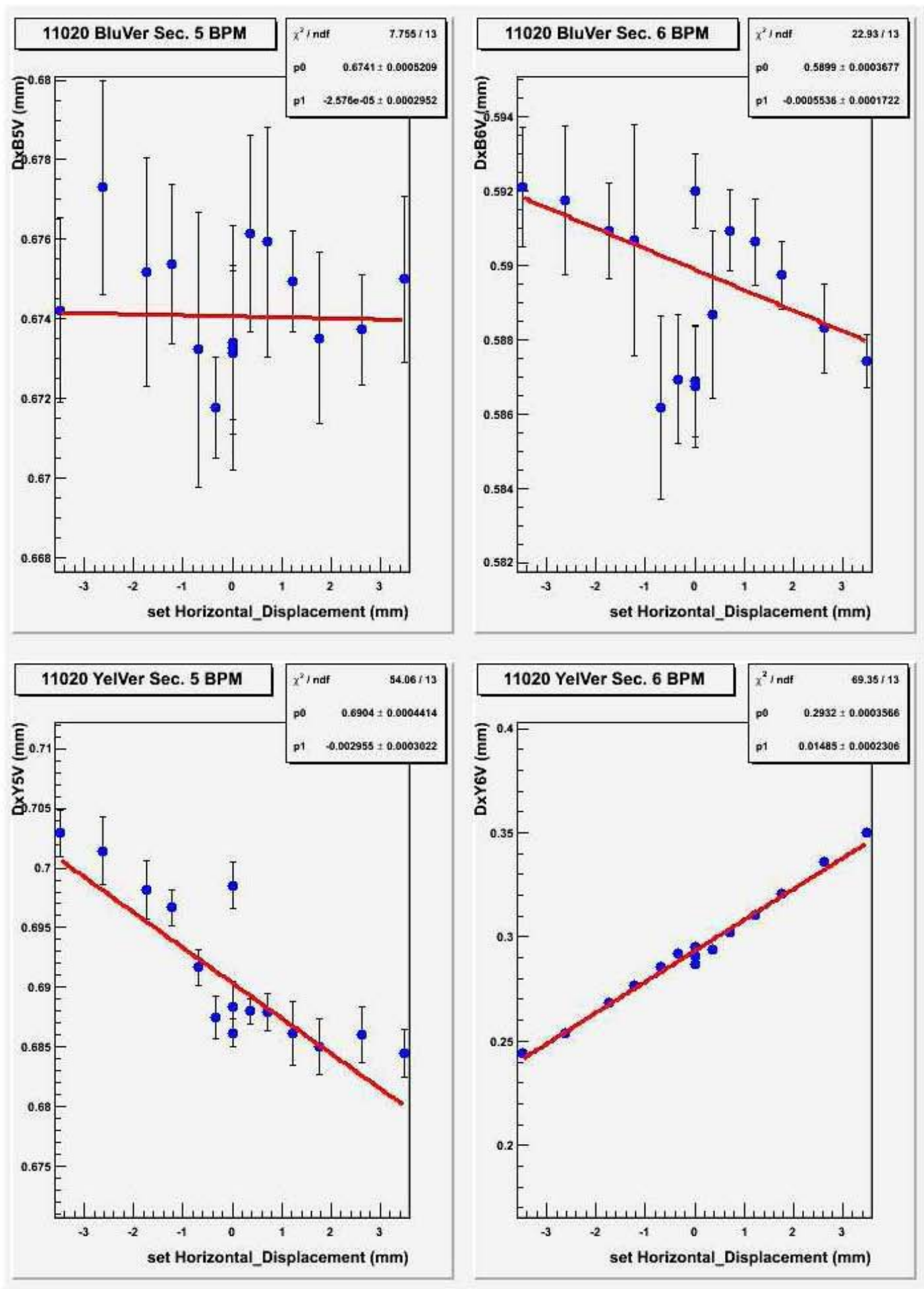

Figure 14: Vertical BPMs during horizontal vernier scan 11020. 

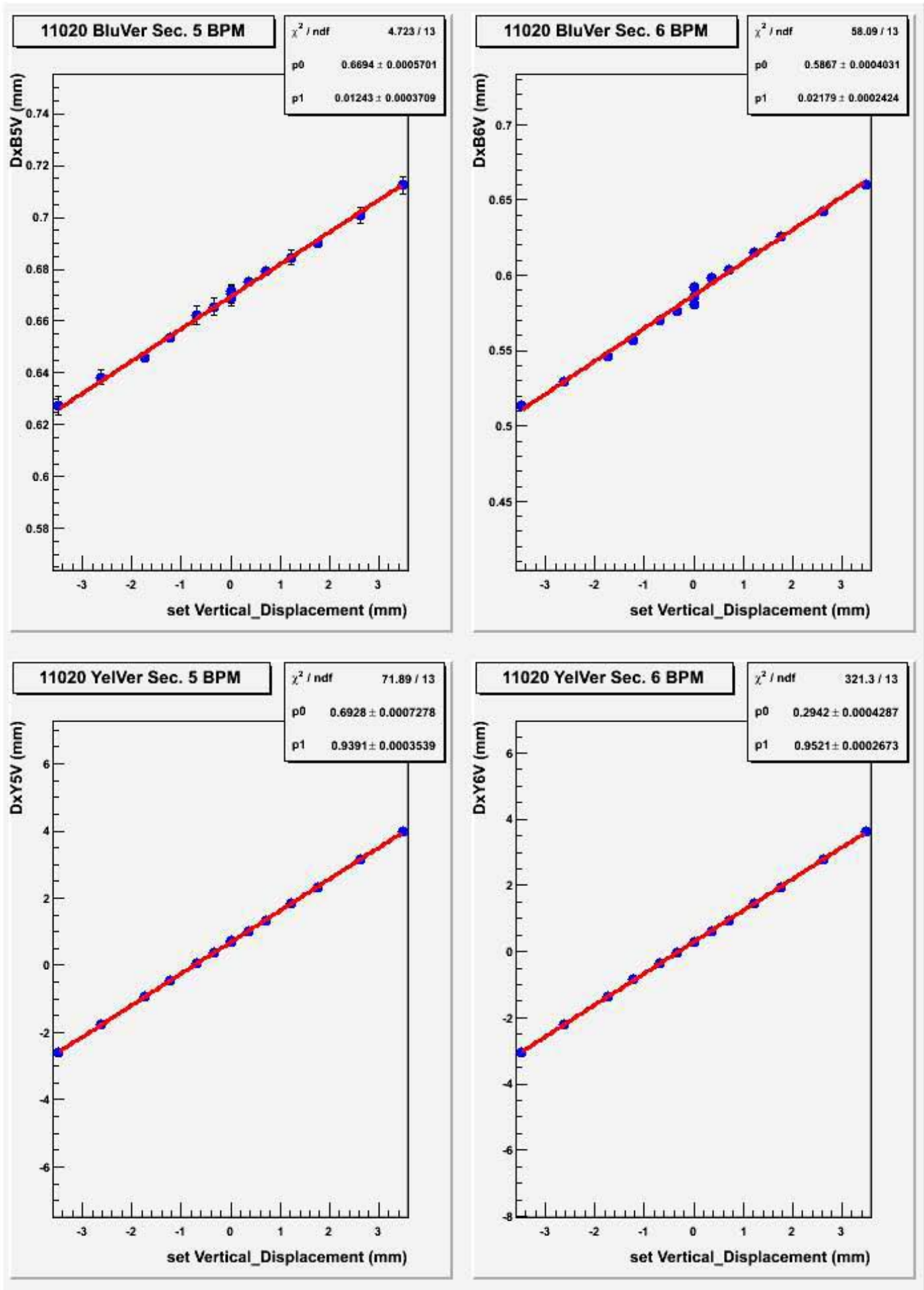

Figure 15: Vertical BPMs during vertical vernier scan 11020. 

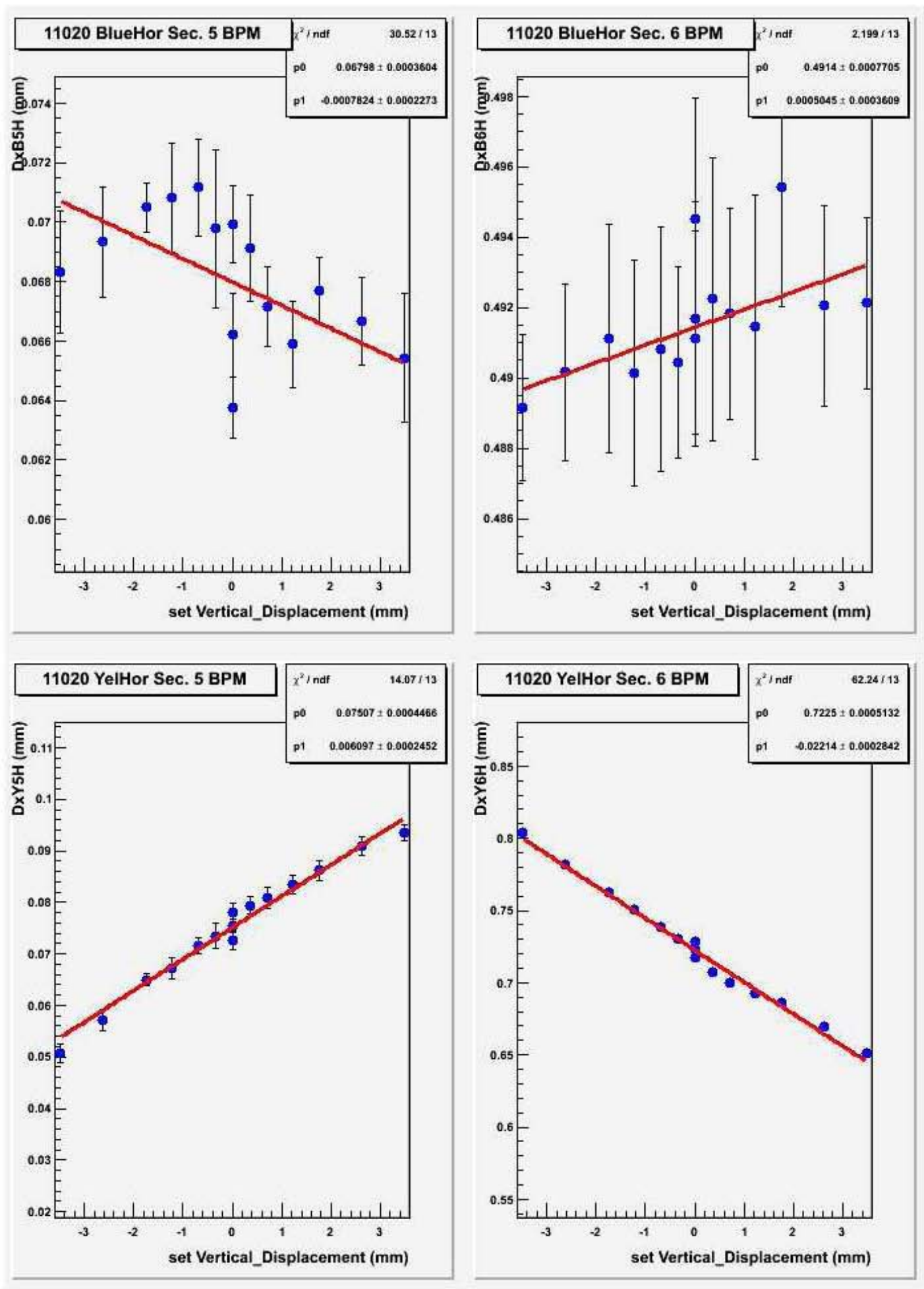

Figure 16: Horizontal BPMs during vertical vernier scan 11020. 

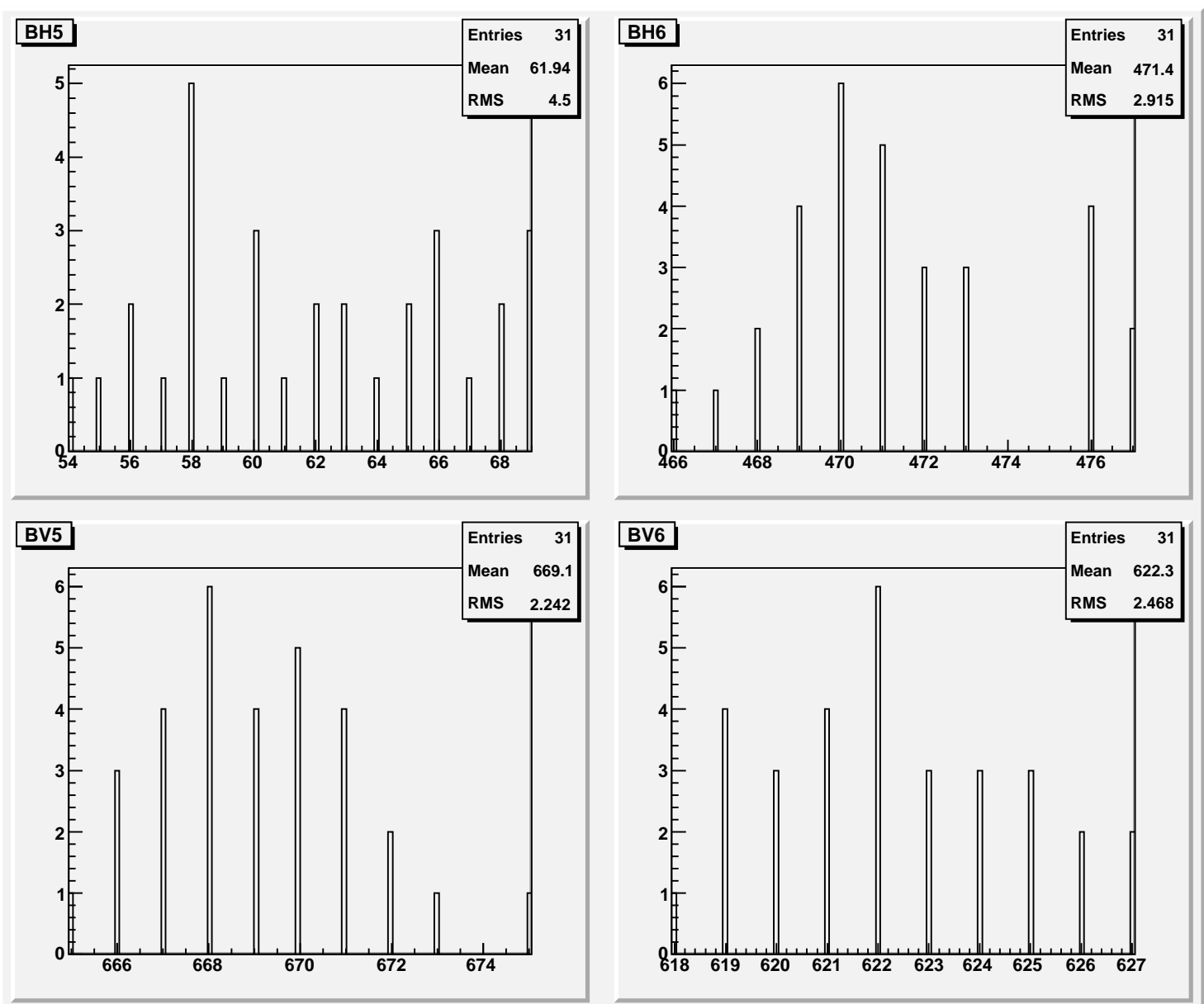

Figure 17: Scatter of blue DX BPM readings (top: horizontal, bottom: vertical) during a randomly chosen 30 minute period in store 11020 . Units are micro meters. 

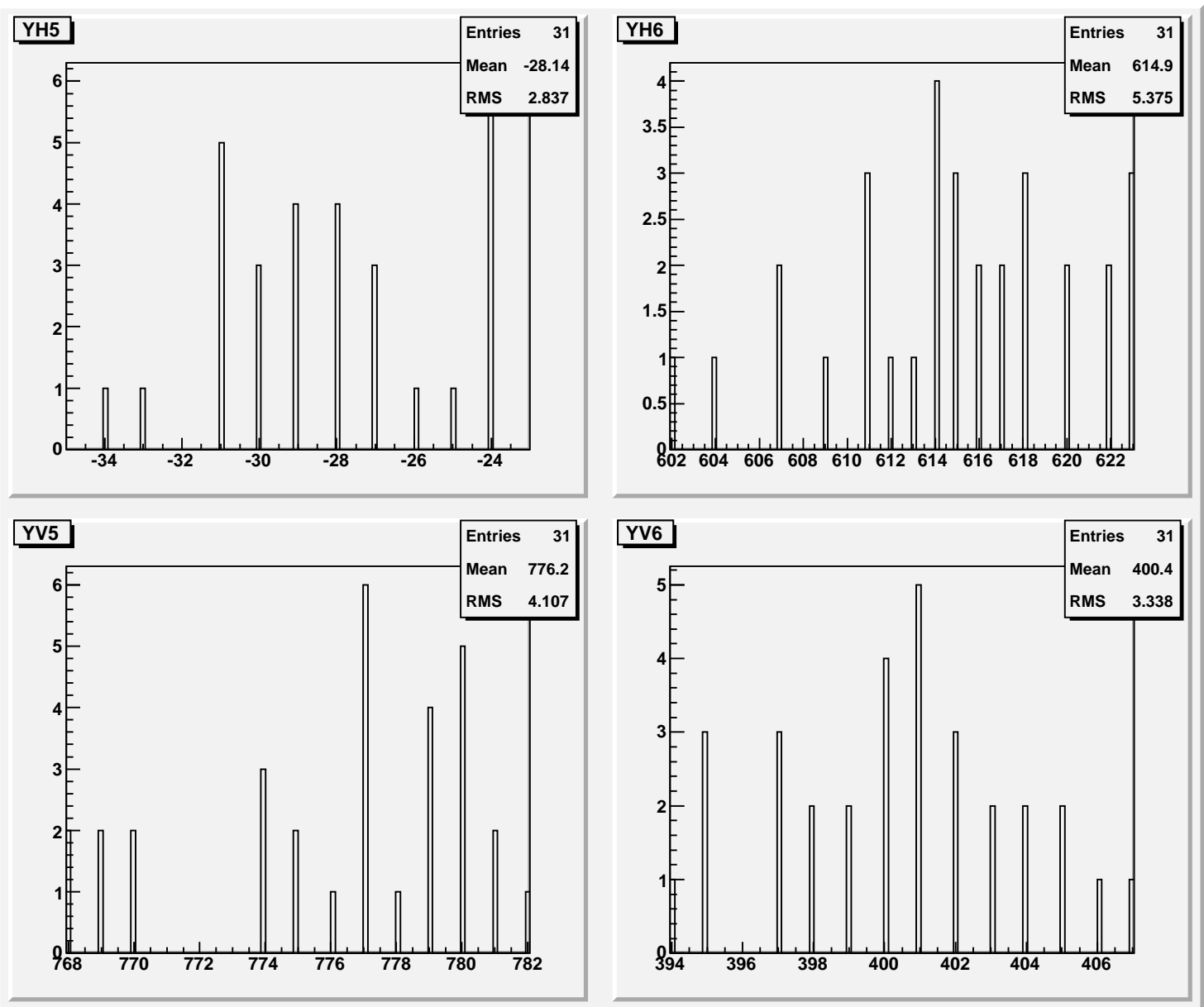

Figure 18: Scatter of yellow DX BPM readings (top: horizontal, bottom: vertical) during a randomly chosen 30 minute period in store 11020. Units are micro meters. 

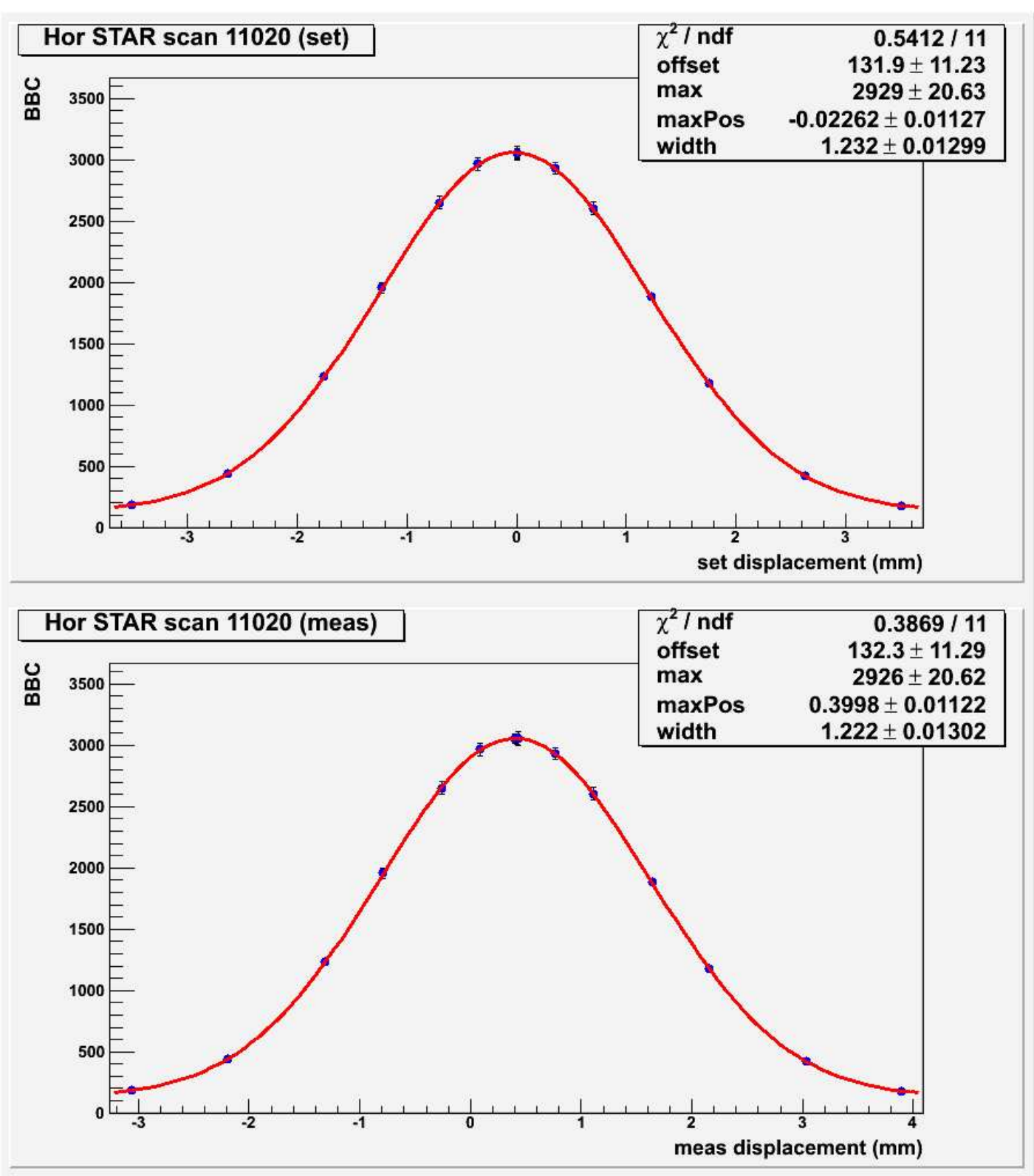

Figure 19: Horizontal vernier scan at IR6 in store 11020. Shown is the collision rate from the $\mathrm{BBC}$ as a function of the displacement using the set values (top) and the measured values (bottom). Only statistical errors are used in the graph. 

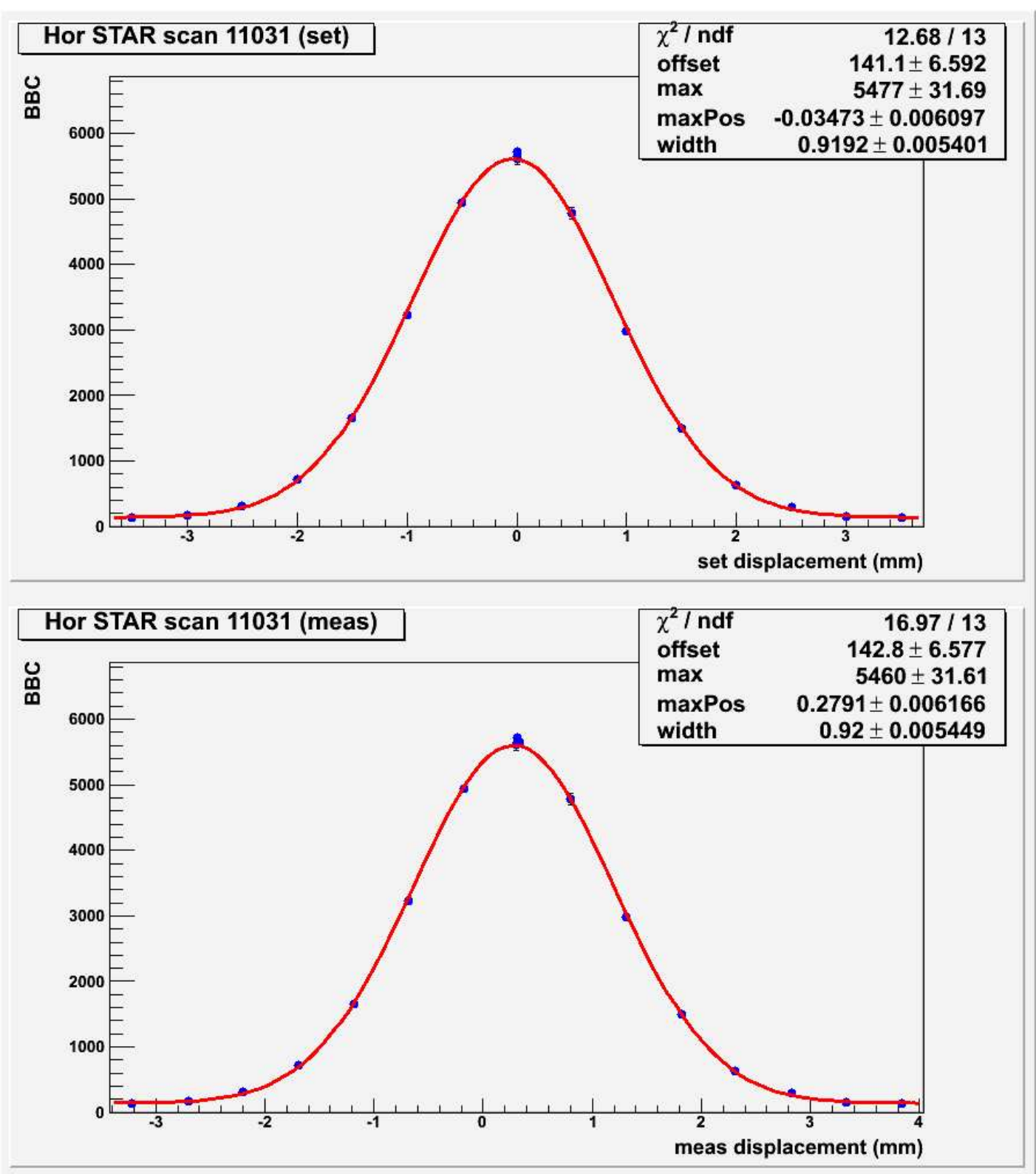

Figure 20: Horizontal vernier scan at IR6 in store 11031. Shown is the collision rate from the $\mathrm{BBC}$ as a function of the displacement using the set values (top) and the measured values (bottom). Only statistical errors are used in the graph. 

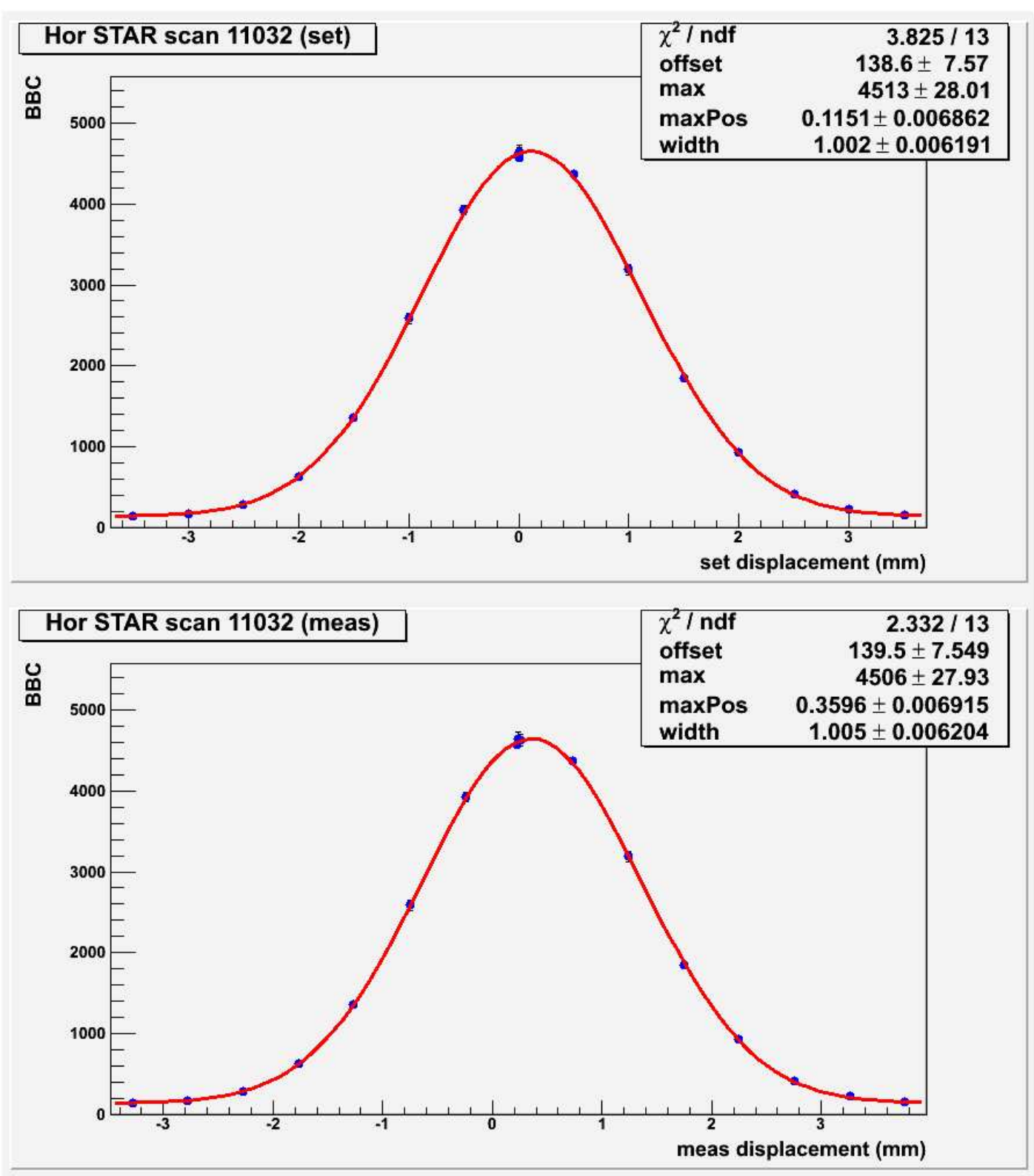

Figure 21: Horizontal vernier scan at IR6 in store 11032. Shown is the collision rate from the $\mathrm{BBC}$ as a function of the displacement using the set values (top) and the measured values (bottom). Only statistical errors are used in the graph. 

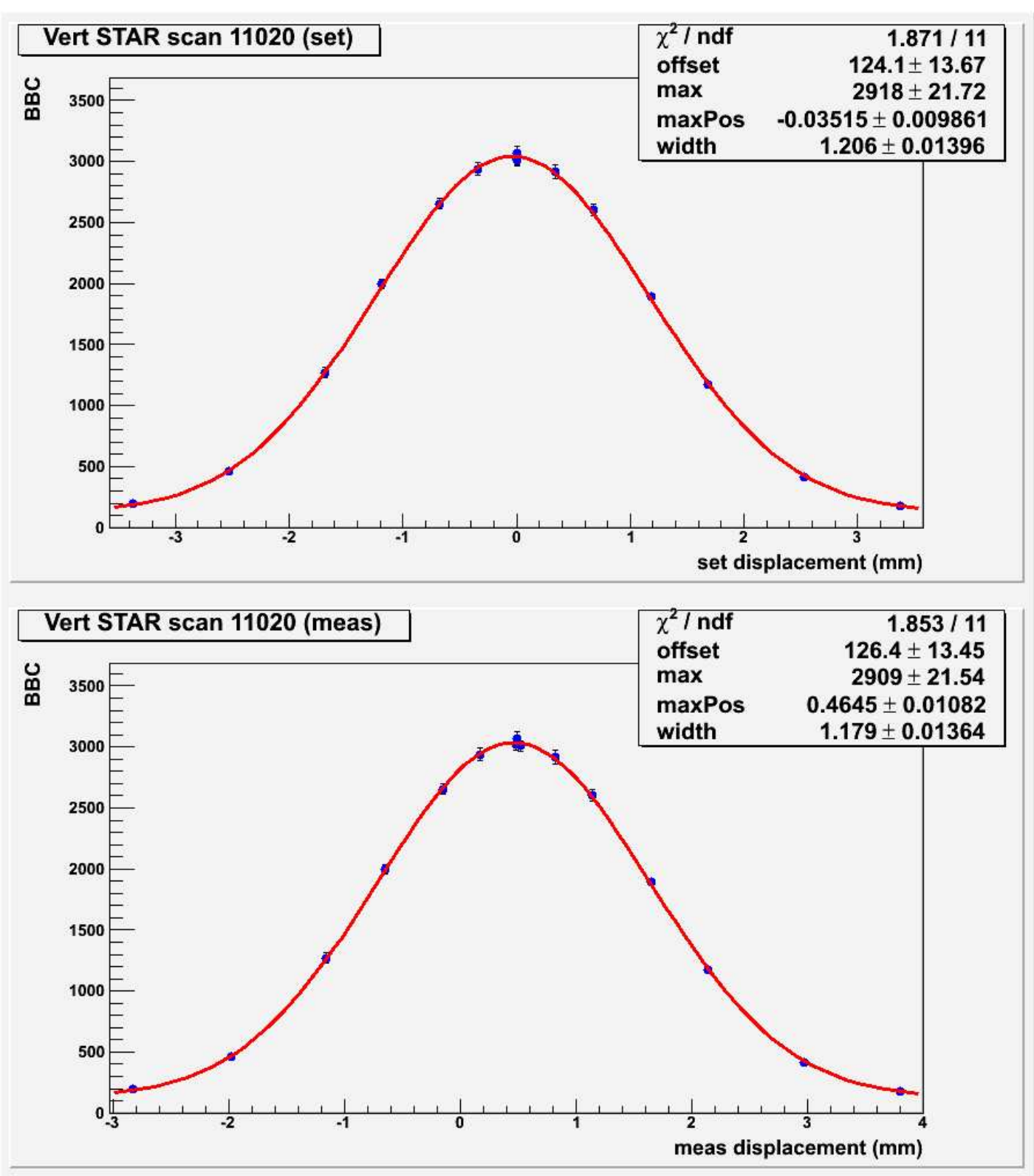

Figure 22: Vertical vernier scan at IR6 in store 11020. Shown is the collision rate from the $\mathrm{BBC}$ as a function of the displacement using the set values (top) and the measured values (bottom). The set values are corrected by a factor 0.964. Only statistical errors are used in the graph. 

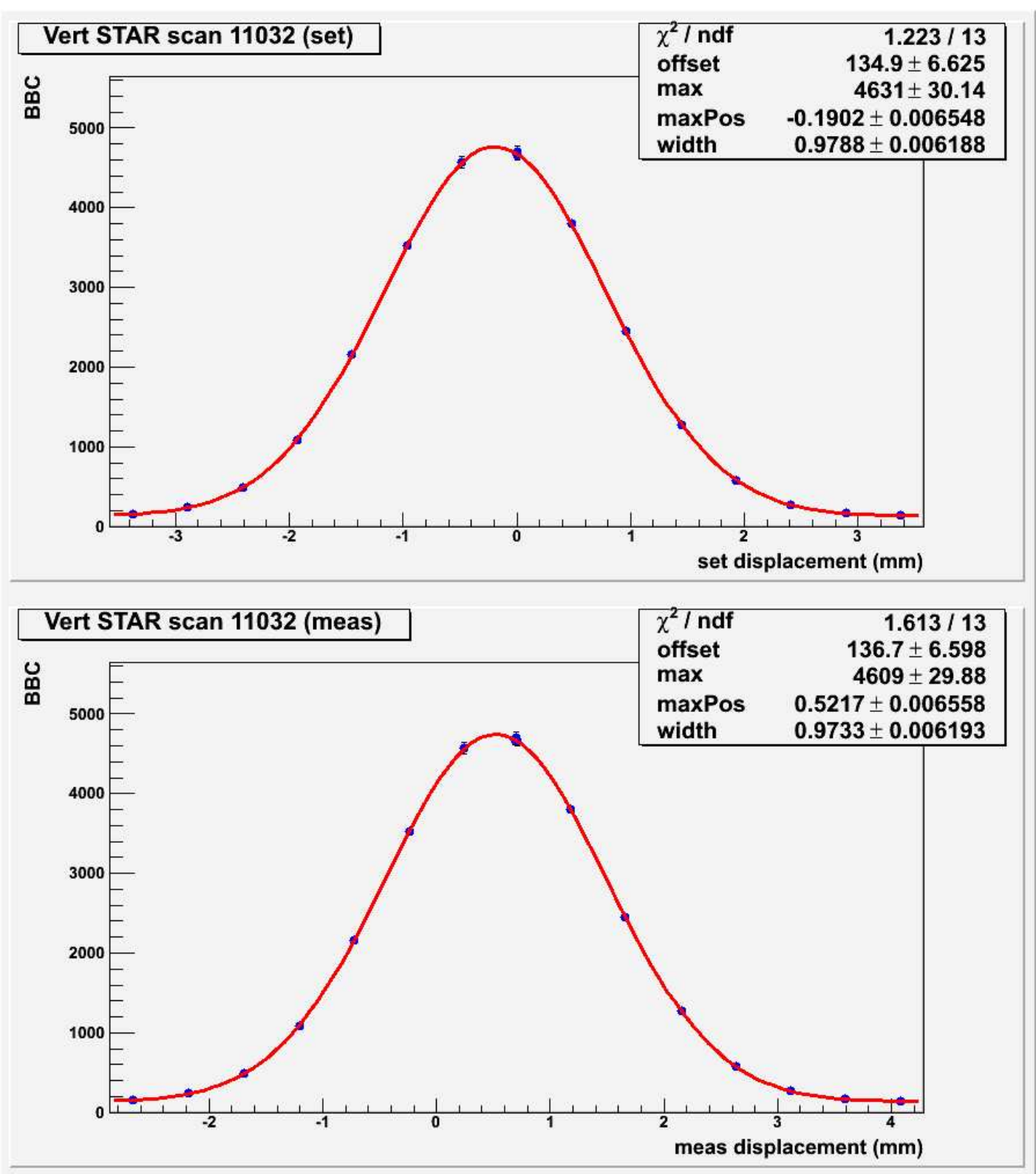

Figure 23: Vertical vernier scan at IR6 in store 11032. Shown is the collision rate from the $\mathrm{BBC}$ as a function of the displacement using the set values (top) and the measured values (bottom). The set values are corrected by a factor 0.964. Only statistical errors are used in the graph. 